\title{
Gattungsgeschichte als transnationale Funktionsgeschichte literarisch-sozialer Institutionen
}

\section{Ein Problemaufriss mit Blick auf den Bildungsroman}

\section{Marcus Twellmann}

Online publiziert: 6 . August 2020

(C) Der/die Autor(en) 2020

Zusammenfassung Der germanistische Ansatz einer Funktionsgeschichte literarisch-sozialer Institutionen ist im Zuge der transnationalen Wende der Literaturwissenschaften zu revidieren. Das Problem wird am Beispiel der Gattung »Bildungsroman« aufgezeigt, deren Entstehung vielfach als eine nationale Sonderentwicklung angesehen wird. Dagegen mehren sich neuerdings Hinweise auf die starke transnationale Verbreitung einer offenbar doch reisenden Form. Wie ließe der Prozess einer national und kulturell grenzüberschreitenden Bewegung sich funktionsgeschichtlich beschreiben?

\section{The history of genres as a transnational functional history of literary and social institutions}

A sketch for the issues with a view on the Bildungsroman

\begin{abstract}
A functional-historical approach that considers literary genres as social institutions has been developed and fruitfully applied in German Studies. The current transnational turn in Literary Studies requires this approach to be revised. The Bildungsroman genre is a distinguished example, as it is widely held to be a national particularity. Recently, though the genre's transnational spread has been pointed out. Obviously, we are dealing with a traveling form. How can we describe its movement across national and cultural boundaries, using a revised functional-historical approach?
\end{abstract}

\footnotetext{
M. Twellmann $(\bowtie)$

Freie Universität Berlin, Habelschwerdter Allee 45, 14195 Berlin, Deutschland

E-Mail: marcus.twellmann@fu-berlin.de
} 


\section{I.}

Das Konzept der »Institution « wird von Vertretern der erstzuständigen Gesellschaftswissenschaft zu den »zentral wichtigen, aber nach wie vor nicht eindeutig geklärten Konzepten $\ll^{1}$ gezählt. Verschiedene Definitionsversuche »umreißen«, so muss das Lexikon Grundbegriffe der Soziologie auch in der jüngsten Auflage berichten, »ein heterogenes Feld, ohne dass sich dabei eine allgemeinverbindliche Kennzeichnung durchgesetzt hätte «. ${ }^{2}$ Im Folgenden soll dieser Begriff mit einem zweiten, ebenso unbestimmten verbunden werden. Man zählt den Funktionsbegriff nämlich »zu den meistgebrauchten, vieldeutigsten und zumeist nur vage oder gar nicht definierten Grundbegriffen der Literaturwissenschaft, der sich je nach Ansatz und Kontext auf ganz unterschiedliche Phänomene beziehen kann «. ${ }^{3}$ Was könnte die Verbindung dieser Grundbegriffe erbringen? Eine nähere Bestimmung beider, die sie für literarhistorische Studien geeignet macht.

Nun liegt eine Theorie der Literaturgeschichte als Funktionsgeschichte von Institutionen bereits vor. Wilhelm Voßkamp hat sie seit den mittleren 1970er Jahren in einer Reihe von Aufsätzen entwickelt. Und so versteht der vorliegende Beitrag sich als eine zunächst theoriegeschichtliche Sichtung und Reflexion. Sie muss Implikationen in einiger Ausführlichkeit entfalten, um im Lichte einer neueren Problem- und Aufgabenstellung, dazu gleich, nicht ausgeschöpfte Potenziale wie auch Hemmnisse ausmachen zu können. Sodann sind neuere soziologische Arbeiten, die von Voßkamp und teils von der Literaturwissenschaft überhaupt noch nicht zur Kenntnis genommen worden sind, für eine Heranführung des literaturwissenschaftlichen Ansatzes an die gegenwärtige Lage heranzuziehen.

Die erwähnten Aufsätze entwerfen, um einige Titel zu zitieren, »Literaturgeschichte als Funktionsgeschichte der Literatur $\aleph^{4}$ und behandeln insbesondere »Gattungen als literarisch-soziale Institutionen $\ll^{5}$ Als Beiträge zur $»$ Romansoziologie $\ll^{6}$ fragen sie in sozialhistorischer Perspektive nach dem Zusammenhang von Gesellschafts- und Gattungsgeschichte vor allem des Bildungsromans. Dabei beschränken sie sich, den Rahmen der germanistischen Disziplin wahrend, auf den deutschsprachigen Raum. Wo ausnahmsweise ein vergleichender Blick geworfen wird, bestätigt sich die Erwartung, dass »eine Institutionalisierung des Bildungsromanmusters, wie

\footnotetext{
1 Hartmut Esser, Soziologie: Spezielle Grundlagen, Bd. 5: Institutionen, Frankfurt a. M. 2000, 1.

2 Roger Häußling, »Institution«, in: Johannes Kopp, Anja Steinbach (Hrsg.), Grundbegriffe der Soziologie, Wiesbaden 2018, 191-193, hier: 193.

3 Ansgar Nünning, »Funktion«, in: Gerhard Lauer, Christine Ruhrberg (Hrsg.), Lexikon Literaturwissenschaft. Hundert Grundbegriffe, Stuttgart 2011, 102-105, hier: $102 \mathrm{f}$.

4 Wilhelm Voßkamp, »Literaturgeschichte als Funktionsgeschichte der Literatur (am Beispiel der frühneuzeitlichen Utopie)«, in: Thomas Cramer (Hrsg.), Literatur und Sprache im historischen Proze $\beta$, Bd. I: Literatur, Tübingen 1983, 32-54.

5 Wilhelm Voßkamp, »Gattungen als literarisch-soziale Institutionen. Zu Problemen sozial- und funktionsgeschichtlich orientierter Gattungstheorie und -historie«, in: Walter Hinck (Hrsg.), Textsortenlehre Gattungsgeschichte, Heidelberg 1977, 27-44.

6 Wilhelm Voßkamp, »Methoden und Probleme der Romansoziologie. Über Möglichkeiten einer Romansoziologie als Gattungssoziologie«, IASL 3 (1978), 1-37.
} 
sie in Deutschland erfolgt, in England nicht stattfindet «. ${ }^{7}$ Mithin verfestigt sich der Eindruck, die »fiktive Darstellung der Bildung eines individuellen Charakters in der konfliktreichen Auseinandersetzung mit der äußeren Realität $\aleph^{8}$ sei eine deutsche Besonderheit. Neuere Befunde sprechen dagegen: »The Bildungsroman, also known as novel of formation or education, is one of the Western forms most profusely used in postcolonial fiction. « ${ }^{9}$ Die veränderte Befundlage sowie die transnationale Wende der Philologien, das ist der Ausgangspunkt der nachstehenden Überlegungen, fordern heute zu einer Revision des Ansatzes heraus. ${ }^{10}$ Möglicherweise könnte die Germanistik damit zu einer Weltliteraturwissenschaft beitragen. Auch die institutionalistische Literaturgeschichtsschreibung rechnet seit jeher mit historischem Wandel. Ist sie aber auf kulturelle Differenz eingestellt und für eine Untersuchung grenzüberschreitender Prozesse geeignet? Neuere soziologische Ansätze, insbesondere jene, die unter dem Namen »Neoinstitutionalismus « firmieren, sind für die Gewinnung einer globalgeschichtlichen Perspektive hilfreich.

\section{II.}

Der Sozialgeschichte dient der Funktionsbegriff dazu, den Gesellschaftsbezug der Literatur zu beleuchten. Die Verwendungsweise entspricht in diesem Zusammenhang jener alltagssprachlichen, die in der Literaturwissenschaft allgemein vorherrscht: Man versteht Funktion als »Aufgabe, Rolle, Leistung oder Wirkung, die ein unselbständiger Teil bzw. ein Element in einem größeren Ganzen hat, spielt bzw. erfüllt«. ${ }^{11}$ Gemäß einem »gemeineuropäischen Sprachgebrauch, der sich bis ins Mittelalter zurückverfolgen läßt «, heißt »Funktion« auch in der Gesellschaftswissenschaft »die einem Teil im Rahmen eines Ganzen obliegende Verrichtung « ${ }^{12}$ Wir haben es offenkundig mit einer teleologischen Denkform zu tun, die in Analogie zum menschlichen Handeln Finalzusammenhänge herstellt. Die Literatur und ihre Elemente, Gattungen etwa, werden als zweckdienliche Teile aufgefasst, die Leistungen für eine größere und höhere Einheit des Sozialen erbringen. Dabei wird das leidliche Funktionieren des Ganzen immer schon unterstellt - ist diese Betrachtungsweise zu retten?

\footnotetext{
7 Wilhelm Voßkamp, »Der Bildungsroman in Deutschland und die Frühgeschichte seiner Rezeption in England «, in: Jürgen Kocka (Hrsg.), Bürgertum im 19. Jahrhundert, Bd. 3, München 1988, 257-286, hier: 273. Siehe zur anglistischen Diskussion Anne-Julia Zwierlein, Der physiologische Bildungsroman im 19. Jahrhundert: Selbstformung, Leistungsethik und organischer Wandel in Naturwissenschaft und Literatur, Heidelberg 2009.

8 Voßkamp (Anm. 5), 36.

9 José Santiago Fernández Vázquez, »Subverting the Bildungsroman in Postcolonial Fiction: Romesh Gunesekera's Reef«, Journal of Postcolonial Writing 36/1 (1997), 30-38, hier: 32.

10 Siehe dazu Marcus Twellmann, »Nationalliteratur als Weltliteratur. Zur Aufgabe der Philologien«, Weimarer Beiträge 64/3 (2018), 360-381.

11 Nünning (Anm. 3), $102 \mathrm{f}$.

12 Niklas Luhmann, »Funktion, IV «, in: Joachim Ritter (Hrsg.), Historisches Wörterbuch der Philosophie, Bd. 2, Basel 1972, Sp. 1142 f., hier: Sp. 1142.
} 
$\mathrm{Zu}$ seiner genauer bestimmten Fassung des Institutionenbegriffs gelangte Voßkamp in der Nachbarschaft von Helmut Schelsky und Niklas Luhmann an der Universität Bielefeld. Schelsky hatte 1970 den Band Zur Theorie der Institution herausgegeben, die erste Publikation des von ihm konzipierten und seit 1968 in Bielefeld ansässigen Zentrums für interdisziplinäre Forschung (ZiF). Der Band ist mit einer ausführlichen Einleitung versehen, die über die Geschichte des Begriffs Auskunft gibt. Daher bezog der Literaturwissenschaftler die Auslegung von Funktion als »Bedürfnissynthese «. Schelsky war dazu anhand der Arbeiten Bronisław Malinowskis gelangt. ${ }^{13}$ Neben Émile Durkheim gilt der aus Polen stammende Anthropologe als Begründer und Namensgeber des »Funktionalismus«, einer Betrachtungsweise von Gesellschaft und Kultur, die, aus dem 19. Jahrhundert stammend, in der ersten Hälfte des 20. Jahrhunderts in verschiedenen Disziplinen lange Zeit dominant war. Vermehrte Kritik an einem »konservativen Funktionalismus, der den sozialen Konflikten gegenüber blind bleibt und lediglich überraschungsfreie Empirie einer theoretischen Stabilitätssuggestion betreibt «, ${ }^{14}$ führte in den 1960 er Jahre zur Verabschiedung.

Wie zu zeigen sein wird, überdauerte der Funktionalismus jedoch sein Ende und ist noch heute, zumal in der Literaturwissenschaft, in hohem Maße wirksam. Ein forschungsgeschichtlicher Rückblick könnte auch aus diesem Grund nützlich sein: Viele Theorieelemente des Funktionalismus sind zu Denkgewohnheiten geworden und werden mitunter auch dort reproduziert, wo die Theorieformation totgesagt wurde und in Vergessenheit geraten ist. Dabei gilt es zu bedenken, dass die Wissenschaften um 1900 ein seit Langem tradiertes, alltägliches Denkmuster aufgriffen und theoretisch reflektierten. Dass dieses Muster die Verabschiedung des Paradigmas auch überdauert, überrascht daher kaum. Auch waren einige Elemente des Funktionalismus längst Gegenstand gelehrter Reflexion. Seit der Antike hat man im Bereich der Poetik und Rhetorik versucht, Gattungen im Hinblick auf ihre Leistung zu bestimmen, was ein Überdauern solcher Denkweisen in der Literaturwissenschaft besonders begünstigt. Hinsichtlich der Anthropologie hat man festgestellt, dass spätere Entwicklungen durch die Auseinandersetzung mit dem Funktionalismus nicht nur angestoßen wurden. Insofern dessen Selbstkritik diese Entwicklungen vorgebahnt habe, könne mit Blick auf die jüngere Fachgeschichte von einer Auflösung der Formation in diverse Ansätze die Rede sein. ${ }^{15}$

Für eine Verknüpfung der Begriffe »Institution« und »Funktion« spricht nicht zuletzt, dass sie dazu anleitet, nach Interdependenzen zu fragen. Vorbildlich war dafür anfangs die Biologie. Nachdem Durkheim im Vorwort zur zweiten Auflage seiner Règles de la méthode die Soziologie als »la science des institutions « ${ }^{16}$ bestimmt

\footnotetext{
13 Helmut Schelsky, »Zur Soziologischen Theorie der Institution«, in: Ders. (Hrsg.), Zur Theorie der Institution, Gütersloh 1970, 9-26, hier: 17.

14 René John, »Funktionale Analyse - Erinnerungen an eine Methodologie zwischen Fixierung und Überraschung «, in: Ders., Anna Henkel, Jana Rückert-John (Hrsg.), Die Methodologien des Systems. Wie kommt man zum Fall und wie dahinter?, Wiesbaden 2010, 29-54, hier: 41.

15 Vgl. Matei Candea, »Severed roots: evolutionism, diffusionism and (structural-)functionalism«, in: Dies. (Hrsg.), Schools and styles of anthropological theory, London 2018, 18-59, hier: 47.

16 Émile Durkheim, »Préface de la seconde Edition«, in: Ders., Les règles de la méthode sociologique, Paris 1927, IX-XXIV, hier: XXIII.
} 
hatte, erklärte sein Schwiegersohn Marcel Mauss 1901 im Artikel »Sociologie« der Grande Encyclopédie: »L'institution est en somme dans l'ordre social ce qu'est la fonction dans l'ordre biologique: et de mème que la science de la vie est la science des fonctions vitales, la science de la société est la science des institutions ainsi définies. ${ }^{17}$ Der vergleichenden Religions- und Rechtsgeschichte verdanke sie die Einsicht, »que certains institutions forment avec certain autres un système«. Daher gelte es, »de ne pas isoler une institution du milieu où elle est apparue«, vielmehr sei die »interdépendance des phénomènes « zu beachten, und zwar in ihrer Dynamik: »Les institutions véritables vivent, c.-a.-d. changent sans cesse «. ${ }^{18}$

Die Anfänge des Funktionalismus liegen weiter zurück. Auguste Comte, der als Erster von sociologie sprach, hatte in die damals neue Wissenschaft auch die Organismus-Analogie eingeführt. Herbert Spencer hatte sodann eine umfangreiche Gesellschaftslehre entwickelt, die das Verhältnis von Teil und Ganzem in stetiger Orientierung an der Wissenschaft vom Leben konzipiert. Daher rührt ein bis heute ungelöstes Problem: Wie ist die Bezugseinheit zu definieren? ${ }^{19}$ Die Biologie hat das Lebewesen zum Gegenstand. Die Geistes-, Gesellschafts- und Kulturwissenschaften können keine vergleichbare Einheit aufweisen. Als sie im 19. Jahrhundert begründet und an staatlichen Universitäten eingerichtet wurden, half ihnen der Nationalismus aus dieser Verlegenheit, indem er die Bezugseinheit unfraglich machte und das fortbestehende Problem damit verdeckte, und zwar mit einer langen Dauer wie sie Institutionen zu eigen ist. Durch die in den 1970er Jahren anhebende Kritik am methodologischen Nationalismus der Soziologie - sie trifft auch die Literaturwissenschaft - wurde dieses Problem auf die Tagesordnung gesetzt, was zu einer schrittweisen, und sei es auch nur schleichenden Veränderung der institutionellen Strukturen beigetragen hat. Die Einrichtung einer Weltliteraturwissenschaft ist als Ziel immerhin vorstellbar.

Spencer fasst die Rede vom sozialen Organismus nicht als bloßes Sprachbild auf, er postuliert eine strukturelle Analogie: Organdifferenzierung und Funktionsteilung zeichne auch die Gesellschaft als ein lebendes Ganzes aus. Der Soziologe besteht darauf, »that in respect of this fundamental trait, a social organism and an individual organism are entirely alike «. ${ }^{20}$ In beiden Fällen beruhe die höhere Einheit auf den stabilen Beziehungen zwischen relativ selbstständigen Teilen oder »Organen«, die sich allerdings prozesshaft veränderten. Die »mutual dependence of parts«, so postuliert Spencer in dem mit »Preparation in Biology « überschriebenen Kapitel von The Study of Sociology, sei »essential for the commencement and advance of social organization, as it is for the commencement and advance of individual organization $\ll{ }^{21}$ Beide sieht er dem gleichen Entwicklungsgesetz der fortschreitenden Differenzierung und Integration unterstellt.

\footnotetext{
17 Marcel Mauss, Paul Fauconnet, »Sociologie«, in: La Grande Encyclopédie, Bd. 30: Sigillateur - Thermopole, Paris 1901, 165-177, hier: 168.

18 Mauss, Fauconnet (Anm. 17), 176 (Hv. i. O.).

19 Vgl. dazu schon Niklas Luhmann, »Funktion und Kausalität«, Kölner Zeitschrift für Soziologie und Sozialpsychologie 14 (1962), 617-644, hier: 629.

20 Herbert Spencer, The Principles of Sociology, Bd. I, 3. Ausg., London, Edinburgh 1893, 452.

21 Herbert Spencer, The Study of Sociology, London 1873, 332.
} 
Als »Organe« des sozialen Organismus werden die Institutionen angesehen. Ihre Funktion soll in der Aufrechterhaltung eines wohl integrierten Ganzen bestehen. Es ist dieser Kerngedanke des Funktionalismus, der in der jüngeren Vergangenheit vermehrt Kritik auf sich gezogen hat, so etwa von Anthony Giddens: »Der Terminus >Funktion< impliziert eine Art teleologischer Qualität, die sozialen Systemen zugeschrieben wird: man hält soziale Gegenstände bzw. Aktivitäten für existent, weil sie funktionalen Erfordernissen entsprechen. «22 Nicht umsonst hatte sich Immanuel Kant noch vor der Übertragung auf weitere Gegenstände bemüht, die Verwendung des Organismusbegriffs auf dem Wege einer transzendentalen Kritik in die Grenzen zu weisen. Allzu schnell gerät die funktionale Analyse zu einer funktionalen Erklärung: Jene höhere Einheit, die vorausgesetzt wird, wo einem vermeintlichen Bestandteil funktionale Notwendigkeit zugeschrieben wird, erscheint als dessen Existenzgrund.

Dabei wird Finalität wohl nur selten im Sinne eines individualistischen Utilitarismus unterstellt, etwa auf dem Wege einer Reduktion auf Absichten von Begründern. Schon Durkheim hatte an den sozio- wie biologischen Grundsatz erinnert, dass die Zwecke, denen ein Organ diene, von den Ursachen seines Daseins unabhängig seien. ${ }^{23}$ Die Funktion ist demnach nicht festgelegt, sondern für Umbesetzungen offen - eine Voraussetzung der institutionengeschichtlichen Betrachtungsweise. Arnold Gehlen kennt eine »sekundäre objektive Zweckmäßigkeit «, ${ }^{24}$ die auf den Ursprung der jeweiligen Institution nicht rückführbar ist. Wo er eine »allgemein durchscheinende großartige Zweckmäßigkeit des organischen Geschehens ${ }^{25}$ konstatiert, da ist eben nicht mehr von der Zwecktätigkeit eines externen Urhebers die Rede. Gemeint ist vielmehr die wechselseitige Bezogenheit und Bestimmung der Teile eines komplexen Ganzen, eine interne Zweckmäßigkeit also. Eine solche Konzeption scheint dem Autor zwingend: »Sobald Religion, Sitte und Recht neutral und als objektive Lebenserscheinungen betrachtet werden, drängt sich die teleologische Denkform auf und es entsteht die Frage nach ihrer Leistung. $\ll^{26}$

Kant hatte, diesem Drang entgegen, Zweckmäßigkeit als regulatives Prinzip einer solchen Betrachtungsweise erläutert, und Nicolai Hartmann hatte die Kritik an der Neigung des Menschen, alles nach der Analogie seiner selbst zu verstehen, fortgeführt: Der Anthropomorphismus bestimme über das mythische Denken hinaus auch die Philosophie und die Naturwissenschaft, wo das forschungsleitende Prinzip als ein konstitutives verkannt und Zweckmäßigkeit am Ende in Zwecktätigkeit verkehrt werde. ${ }^{27}$ Auch Hartmann aber wollte dem teleologischen Denken nicht ein Ende machen, sondern Zweckmäßigkeit philosophisch als eine Fiktion erhellen, die für

\footnotetext{
22 Anthony Giddens, Die Konstitution der Gesellschaft. Grundzüge einer Theorie der Strukturation, Frankfurt a. M., New York 1997, 350.

$23 \mathrm{Vgl}$. Durkheim, Les règles de la méthode (Anm. 16), 113.

24 Vgl. Arnold Gehlen, Der Mensch. Seine Natur und seine Stellung in der Welt, in: Ders., Gesamtausgabe, Bd. 3.1, hrsg. Karl-Siegbert Rehberg, Frankfurt a. M. 1993, 472-480.

25 Gehlen (Anm. 24), 77.

26 Gehlen (Anm. 24), 455 (Hv. i. O.).

27 Vgl. Nicolai Hartmann, Philosophie der Natur. Abriss der speziellen Kategorienlehre, Berlin 1980, v. a. $672-713$.
} 
die Untersuchung organischer Gefüge notwendig ist. ${ }^{28}$ Von diesem Stand ausgehend wäre die Funktionalismus-Diskussion heute fortzusetzen.

Indessen lässt das Zugeständnis der Notwendigkeit einer Als-ob-Betrachtung den anderen gegen-funktionalistischen Einwand bestehen: Die synchrone Analyse gesellschaftlicher Zusammenhänge unterliegt vielfach dem Eindruck eines statischen Gleichgewichtszustands, zu dessen Erhaltung die Institutionen eines als Organismus gedachten Kultur- oder Gesellschaftsganzen vermeintlich dienen. Daraus erhellt der konservative Zug dieser Betrachtungsweise - kaum zufällig trägt Schelskys erster, 1949 nach einem Weltkrieg vorgelegter Versuch in dieser Sache den Titel Über die Stabilität von Institutionen. ${ }^{29}$

Seit 1947 hatte Schelsky gemeinsam mit Arnold Gehlen in der Karlsruher American Library Schlüsselwerke der US-amerikanischen Forschung studieren können und sich damit gegenüber deutschen Fachkollegen einen Vorsprung verschafft. ${ }^{30}$ Bei Malinowski fand er jene Begriffsauslegung vor, auf der Voßkamps Verständnis von Institutionen als »Bedürfnissynthesen« beruht. Malinowski, der mit Argonauts of the Western Pacific (1922), dem Bericht von seinen Aufenthalten auf den Trobriand-Inseln, das Paradigma der teilnehmenden Beobachtung begründete, nahm die Perspektive des Feldforschers ein, als er 1926 im Eintrag »Anthropology« der Encyclopedia Britannica die »Functional Analysis of Culture « erläuterte: »He [the fieldworker, Anm. MT] perceives that culture, above all, provides primitive man with the means of satisfying his wants, and of mastering his surroundings. The functional view of culture insists therefore upon the principle that in every type of civilisation, every custom, material object, idea and belief fulfils some vital function, has some task to accomplish, represents an indispensable part within a working whole. $\ll^{31}$ Wie Mauss so betont auch Malinowski, es gelte nicht einzelne kulturelle Objekte, sondern »complexes of such objects $«{ }^{32} \mathrm{zu}$ untersuchen, d.h. Kulturen, die Menschengruppen die Selbstbehauptung - hier wird das darwinistische Erbe greifbar unter den jeweils gegebenen Umweltbedingungen erlauben. ${ }^{33}$

Ausführlich hat Malinowski in A Scientific Theory of Culture (1944) die Hauptbestandteile einer Kultur als Institutionen und den Gegenstand seiner Disziplin dementsprechend bestimmt: »Culture is an integral composed of partly autonomous, partly coördinated institutions $« ;{ }^{34} \gg$ scientific anthropology consists in a theory of

\footnotetext{
28 Vgl. Nicolai Hartmann, Teleologisches Denken, Berlin 1951, 10.

29 Helmut Schelsky, »Über die Stabilität von Institutionen, besonders Verfassungen. Kulturanthropologische Gedanken zu einem rechtssoziologischen Thema« [1949], in: Ders., Auf der Suche nach Wirklichkeit, Düsseldorf, Köln 1965, 33-55.

30 Vgl. Karl-Siegbert Rehberg, »Vom soziologischen Neugründungs-Pragmatismus zur >Anti-Soziologie $<$. Helmut Schelskys Position in der Nachkriegsgeschichte des Faches «, in: Alexander Gallus (Hrsg.), Helmut Schelsky - der politische Anti-Soziologe. Eine Neurezeption, Göttingen 2013, 17-36, hier: 18.

31 Bronisław Malinowski, »Anthropology«, in: Encyclopedia Britannica, London, New York 1926, 131-140, hier: 133 .

32 Malinowski (Anm. 31), 138.

33 »The real identities of culture appear to lie in the organic connection of its parts, in the function which a detail fulfils within its scheme, in the relation between the scheme, the environment and the human needs. « Malinowski (Anm. 31), 139.
}

34 Bronisław Malinowski, A Scientific Theory of Culture, Chapel Hill 1944, 40. 
institutions, that is, a concrete analysis of the type units of an organization «. ${ }^{35}$ In diesem Zusammenhang wird »Funktion« wie folgt bestimmt: »the satisfaction of a need by an activity in which human beings coöperate, use artifacts, and consume goods $\ll{ }^{36}$ Ersichtlich zielt diese Definition weniger auf die Leistung einer Institution für das Kulturganze als auf »the satisfaction of the organic or basic needs of man «. ${ }^{37}$ Um eine literaturwissenschaftliche Relevanz dieser im engen Wortsinn anthropologischen Konzeption in Aussicht zu stellen, sei schon hier hinzugefügt, dass sie auch voraussetzungsreichere Funktionen, ästhetische etwa, umfasst, die durch die institutionelle Dauerbefriedigung basaler Bedürfnisse erst möglich gemacht würden: »Culture thus satisfies first the organic standard of living and then adds an increased artificial standard of enjoyment, in which aesthetic pleasures, joys of companionship; and creative achievements can be developed. In all this, culture is an organic unit $\ll^{38}$ - eine höhere Einheit, die aber der Befriedigung menschlicher Bedürfnisse diene.

Indem Malinowski, so erläutert Schelsky, »den Institutionen die Leistung der sozialen Integration der auf Bedürfnisse bezogenen Funktionen zuschreibt, erklärt er den Systemcharakter der Gesellschaft aus Funktion und Institution, und nicht umgekehrt. Seine Vorgegebenheit ist >der Menschく, nicht >die Gesellschaft< oder $>$ die Kultur $<.{ }^{39}$ Der Anthropologe hatte zunächst biologische Grundbedürfnisse wie die Ernährung im Blick. Diese Betrachtungsweise gewinnt in der Gegenüberstellung mit jener ihr Profil, die bei den höheren Ganzheiten ansetzt. Radcliffe-Brown, der sich auf Durkheim berufen kann, ${ }^{40}$ ersetzt $»$ needs « durch $»$ necessary conditions of existence « ${ }^{41}$ um die Funktion auf die Gesellschaft zu beziehen ${ }^{42}$ Schelsky war um eine Vermittlung der unterschiedlichen Sichtweisen bemüht. ${ }^{43} \mathrm{Zu}$ seiner Zeit sah

35 Malinowski (Anm. 34), 41.

36 Malinowski (Anm. 34), 39.

37 Malinowski (Anm. 34), 37.

38 Bronisław Malinowski, »The Pan-African Problem of Culture Contact«, American Journal of Sociology 48/6 (1943), 649-656, hier: 651.

39 Schelsky (Anm. 13), 15.

40 »La fonction d'un fait social ne peut être que sociale, c'est à-dire qu'elle consiste dans la production d'effets socialement utiles. Sans doute, il peut se faire, et il arrive en effet, que, par contre-coup, il serve aussi à l'individu. Mais ce résultat heureux n'est pas sa raison d'être immédiate. Nous pouvons donc compléter la proposition précédente en disant: La fonction d'un fait social doit toujours être recherchée dans le rapport qu'il soutient avec quelque fin social.« (Émile Durkheim, Les règles de la méthode sociologique, Paris 1895 , 135.).

41 Alfred Reginald Radcliffe-Brown, Structure and Function in Primitive Society. Essays and Addresses, Glencoe 1952, 178.

42 »As the word function is here being used the life of an organism is conceived as the functioning of its structure. It is through and by the continuity of the functioning that the continuity of the structure is preserved. If we consider any recurrent part of the life-process, such as respiration, digestion, etc., its function is the part it plays in, the contribution it makes to, the life of the organism as a whole.« RadcliffeBrown (Anm. 41), 179.

43 Neben der »Darstellung einer differenzierten Sozialstruktur« sei »die soziologische Vermittlung von Individuum und Gesellschaft« eine zweite Zielsetzung des Institutionenbegriffs bei Spencer gewesen, so Helmut Schelsky, »Die Institutionenlehre Herbert Spencers und ihre Nachfolger «, in: Ders., Die Soziologen und das Recht. Abhandlungen und Vorträge zur Soziologie von Recht, Institution und Planung, Opladen 1980, 248-261, hier: 252. 
er sich vor allem durch die Systemtheorie Niklas Luhmanns zu einer Konzeption herausgefordert, die menschliches Handeln mit Systemstrukturen in Verbindung hält.

Wohlgemerkt wollte Malinowski kulturelle Erscheinungen nicht auf biologische Ursachen reduzieren, ${ }^{44}$ das betont Schelsky bereits in seinem frühen Aufsatz. ${ }^{45}$ Dem kulturellen Umweg der Befriedigung gilt das eigentliche Interesse. Der Anthropologe erkannte, dass kulturelle Einrichtungen auch abgeleitete Bedürfnisse freisetzen, die wiederum nach Befriedigung verlangen. Von »Synthesen « ist deshalb die Rede, weil Institutionen auf eine Pluralität sowohl biologischer Primärbedürfnisse als auch kultureller Sekundärbedürfnisse zu beziehen sind. Sie sind, wie mit Sigmund Freud gesagt wurde, »überdeterminiert «. ${ }^{46}$ In der einen wie in der anderen Hinsicht kann nach ihrer Funktion gefragt werden, das ist Schelsky wichtig und bahnt einer literaturwissenschaftlichen Aneignung dieser Betrachtungsweise den Weg: »Zur Selbsterhaltung des Menschen oder der Gruppe dienen sie alle, das ist kein Unterschiedskriterium für biologische und kulturelle Bedürfnisse beim Menschen. Die Erfüllung kultureller Bedürfnisse kann genauso als >Funktion< aufgefaßt werden wie die der biologischen Primärbedürfnisse. $\ll^{47}$

Um das Element der bedürfniserzeugenden Wirkung hat Voßkamp seine Theorie, mit Verweis auf Schelsky, erst später ergänzt. ${ }^{48}$ Die früheren Aufsätze dokumentieren das Bestreben, literarische Formen als Antworten auf vorgegebene Probleme zu beziehen. Die anthropologische Sicht auf das Institutionelle erwies sich einer rezeptionsästhetisch informierten Romansoziologie auch deshalb als verwandt, weil deren Vorgegebenheit ebenfalls »der Mensch« war, genauer: »der Leser«: »Kommunikativer Erfolg und > Wirkung < beim Rezipienten sind weitgehend davon abhängig, inwieweit der Leser durch den Roman >eine Befriedigung seiner gegenwärtigen Bedürfnisse erfährt $<. \ll^{49}$ Eben darin erkennt die Funktionsgeschichte den Grund für die erfolgreiche Institutionalisierung von Gattungen: dass sie »Möglichkeiten (zeitlich begrenzter) Bedürfnisbefriedigung für bestimmte Leserschichten eröffnen. Insofern sind Gattungen >Bedürfnissynthesen<, in denen nicht nur bestimmte Problemlagen artikuliert, sondern auch Lösungsstrategien diskutiert und angeboten werden. $\ll^{50}$

Die Problemlagen aber, in denen lesende Menschen nach Lösungen fragen, sollten aus der Gesellschaftsgeschichte resultieren. Es gibt wohl keine Gattung, an der die Bezogenheit auf ein nationales Ganzes sich besser zeigen ließe als eben den Bildungsroman. Voßkamps These lautet, die Konzeption des Bildungsromans sei begreifbar »aus der politisch-sozialen Funktion, die diese spezifische Zeitutopie

\footnotetext{
44 Vgl. Malinowski (Anm. 34), 89.

45 »[D]a das Wesen der Erfüllung dieser Bedürfnisse gerade darin besteht, daß der Mensch die sie befriedigende Wirklichkeit selbst schafft, dauernd erneuert und aufrechterhält, so kann man den Prozeß der Befriedigung dieser Bedürfnisse zugleich von seiner anderen Seite her als den Schöpfungsprozeß der Kultur bezeichnen.« Schelsky (Anm. 29), 43.

46 Vgl. Schelsky (Anm. 13), 19.

47 Schelsky (Anm. 13), 18.

48 Bei der Untersuchung literarischer Gattungen seien auch »Funktionen des Freisetzens und Erzeugens neuer Bedürfnisse « zu berücksichtigen. Voßkamp (Anm. 4), 40 (Hv. i. O.).

49 Voßkamp (Anm. 6), 33.

50 Voßkamp (Anm. 4), 40.
} 
als identitätsstiftende, politische Defizite ausfüllende literarisch-soziale Institution in Deutschland übernimmt $«{ }^{51}$ Es waren demnach hoch besondere, durch eine eigentümliche Nationalgeschichte bedingte Bedürfnisse, denen diese Erzählform entsprach.

Eine Revision dieser These muss das traditionell starke Interesse der deutschen Germanistik an dieser Gattung bedenken. Zu Zeiten wurde mit unverhohlenem Nationalstolz ein Besitzanspruch erhoben: »Seit Goethe besitzen wir«, so formuliert etwa Herman Anders Krüger im Jahr 1906, »eine Romanart, die ein ganz ausgesprochen nationales Gepräge trägt, wie sie eigenartiger, individueller kein anderes Volk aufzuweisen hat, den deutschen Bildungsroman. $\ll^{52}$ Der Anspruch umfasst auch den Bildungsgedanken. Die von Herman Nohl zur gleichen Zeit begründete Vorstellung von einer »deutschen Bewegung « ist in der Historiografie und im Selbstverständnis der Pädagogik in Deutschland bis heute wirksam. ${ }^{53}$

Auch unabhängig von manifest politischen Motiven ist - aus der nötigen Distanz - eine Tendenz zur nationalen Partikularisierung zu erkennen: »The Germanist is likely to insist that the Bildungsroman develops in a particular political and cultural climate in Germany«, so der Amerikaner Todd Kontje, »and that its history in critical discourse remains intimately linked to the shifting fortunes of German history. In contrast, those outside of German studies downplay the national connection and stress the genre's close ties to modernity in general. « ${ }^{54}$ Wohl zeigt der Gebrauch der deutschsprachigen Bezeichnung »Bildungsroman « in anderssprachigen Texten, dass man den Ursprung allgemein in Deutschland verortet; auch über die Musterfunktion von Goethes Wilhelm Meisters Lehrjahre (1795/96) herrscht weitgehend Einigkeit. Dennoch ist das mythologische Moment einer germanistischen Literaturgeschichtsschreibung zu beachten, die das nationale Selbstverständigungsnarrativ eines »deutschen Sonderwegs« forterzählt.

Wie alle Erzählungen dieser Art so behauptet auch diese Einzigartigkeit - »there is nothing unusual about German exceptionalism $\ll .{ }^{55}$ Identitätsbildend wirkt sie auch in ihren kritischen Varianten, ja noch in jener eines » deutschen Sonderwegs $<$ in den Abgrund « des Nationalsozialismus. ${ }^{56}$ Wo Thomas Mann den Bildungs- und Entwicklungsroman als »typisch-deutsch, legitim national« bezeichnet, zielt er bekanntermaßen auf einen »deutschen Humanitätsbegriff«, der »das Produkt einer Epoche ist, in der die Gesellschaft in Atome zerfiel und die aus jedem Bürger einen Menschen

\footnotetext{
51 Voßkamp (Anm. 5), 37 (Hv. i. O.).

52 Herman Anders Krüger, »Der neuere deutsche Bildungsroman«, Westermanns Monatshefte 51. Jg., Bd. 101, 1. Teil, 1906, 257-272, hier: 270 (Hv. i. O.).

53 Vgl. Jürgen Oelkers, »Das Konzept der Bildung in Deutschland im 18. Jahrhundert«, Zeitschrift für Pädagogik 38 (1998), 45-70, hier: 65-67.

54 Todd Kontje, The German Bildungsroman: History of a National Genre, Columbia 1993, 111. Letzteres gilt etwa für Franco Moretti, The way of the world: The Bildungsroman in European Culture, London 1987.

55 James Sheehan, »Paradigm Lost? The >Sonderweg< Revisited«, in: Gunilla Budde, Sebastian Conrad, Oliver Janz (Hrsg.), Transnationale Geschichte. Themen, Tendenzen und Theorien, Göttingen 2006, 150-161, hier: 150.

56 Hans-Ulrich Wehler, »>Deutscher Sonderweg< oder allgemeine Probleme des westlichen Kapitalismus?«, Merkur 35/5 (1981), 478-487, hier: 478.
} 
machte, das politische Element von jeher fast völlig fehlte «. ${ }^{57}$ Manns Ausführungen über »die vielleicht berühmteste Eigenschaft der Deutschen, diejenige, die man mit dem sehr schwer übersetzbaren Wort >Innerlichkeit< bezeichnet «, ${ }^{58}$ stehen im Einklang mit jenen Helmuth Plessners über die »verspätete Nation «. ${ }^{59}$ Kurz gesagt: In Deutschland, wo das Bürgertum es unterließ, auf dem Wege einer Revolution die politische Macht zu erringen, bemühten die Angehörigen dieser gesellschaftlich gleichwohl aufstrebenden Schicht sich stattdessen um die Entfaltung ihrer Individualität. Ohne die genannten Stichwortgeber zu zitieren, spricht auch Voßkamp von einer »Wendung ins bloß Private«, von einer »melancholisch-weltfluchtartigen Verinnerlichung und Resignation $« .{ }^{60}$ Wohl ist dieser Erzählung, ihres nationalmythologischen Charakters ungeachtet, ein gewisser Realitätsgehalt zuzugestehen. Die exzeptionalistische Überakzentuierung von Besonderheit im Sinne von Einzigartigkeit und folglich Unvergleichbarkeit verstellt aber, darauf kommt es hier an, den Blick auf das Gemeinsame verschiedener Nationen.

Größeres Gewicht als der nationalen Geschichte legt Voßkamp, recht besehen, einem gesellschaftsgeschichtlichen Prozess der zunehmenden Arbeitsteilung und funktionsorientierten Differenzierung bei. Dieser Vorgang sei mit einer krisenhaften Erfahrung der radikalen Autonomisierung und Zersplitterung verbunden gewesen; die Deutschen seien ihr mit der utopischen Vorstellung des allseitig gebildeten Individuums begegnet. ${ }^{61}$ Mit der Veränderung von Strukturen ist jedoch ein wesentlicher Zug dessen angesprochen, was auch außerhalb der Germanistik »gesellschaftliche Moderne « heißt und als Entstehungsbedingung der Gattung angeführt wird. In welchem Maße Deutschland sich durch eine außergewöhnliche Wertschätzung für die allseitige Ausbildung menschlicher Fähigkeiten von anderen Nationen unterscheidet, bleibt genauer zu untersuchen. Hier nur so viel: Dass die Allgemeine Erklärung der Menschenrechte des Jahres 1948 in Artikel 26 jedem Menschen ein - so zumindest der deutsche Wortlaut - »Recht auf Bildung « zuspricht und genauerhin »die volle Entfaltung der menschlichen Persönlichkeit« als deren Ziel deklariert, weist auf eine weite Verbreitung dieses Werts hin. Mit der deutschen Tradition - Joseph Slaughter, darauf wird zurückzukommen sein, hat das überzeugend dargelegt -, steht der Bildungsbegriff der Vereinten Nationen, auch dort, wo von education, éducation, educación, istruzione oder ögrenim die Rede ist, in einer hohen Kontinuität. ${ }^{62}$

\footnotetext{
57 Thomas Mann, »Der autobiographische Roman«, in: Ders., Gesammelte Werke in 13 Bänden, Bd. XI: Reden und Aufsätze 3, 2., durchges. Aufl., Frankfurt a. M. 1974, 700-703, hier: 702.

58 Thomas Mann, »Deutschland und die Deutschen«, in: Ders. (Anm. 57), 1126-1148, hier: $1141 \mathrm{f}$.

59 Vgl. Helmuth Plessner, Die verspätete Nation, Frankfurt a. M. 1974, 14.

60 Wilhelm Voßkamp, »Gattungen«, in: Helmut Brackert, Jörn Stückrath (Hrsg.), Literaturwissenschaft. Ein Grundkurs, Reinbek bei Hamburg 1992, 253-269, hier: 262 f.

61 »Die >Zweckfreiheit< bildungsbürgerlicher Kultur bietet ein kritisches und/oder kompensatorisches Gegenüber zur utilitaristischen Ökonomie und zweckrationalen Politik. « Wilhelm Voßkamp, »Der Bildungsroman als literarisch-soziale Institution. Begriffs- und funktionsgeschichtliche Überlegungen zum deutschen Bildungsroman am Ende des 18. und Beginn des 19. Jahrhunderts «, in: Christian Wagenknecht (Hrsg.), Zur Terminologie der Literaturwissenschaft, Stuttgart 1989, 337-352, hier: 348.

62 Vgl. Joseph R. Slaughter, Human Rights, Inc.: The World Novel, Narrative Form, and International Law, New York 2007.
} 
Jedenfalls ist der Bildungsroman ein besonders gut geeignetes Beispiel, vielleicht das beste, für die hier zu entfaltende Problematik: Wenn die Gattung mit anderen gesellschaftlichen Institutionen einer Nation dermaßen eng zusammenhängt, wie ist dann ihre grenzüberschreitende Mobilität zu beschreiben, hinsichtlich ihrer Funktion zu begreifen oder gar zu erklären? Dass funktionalistische Institutionengeschichte in diachroner Perspektive mit Wandel rechnet, ist ein erster Ansatzpunkt. Das statische Äquilibrium, das man vor allem in »traditionalen«, ebenso aber in »modernen« Gesellschaften erkennen wollte, war stets ein Theorieartefakt. Daher war die Dynamisierung der Konzeption ein notwendiger Schritt. Schelsky benutzte schon früh den Prozessbegriff »Institutionalisierung « und so auch Voßkamp. ${ }^{63}$ Nichtsdestotrotz galt das primäre Interesse der Etablierung der Gattung im Sinne eines »Auskristallisierens, Stabilisierens und institutionellen Festwerdens von dominanten Strukturen $\ll{ }^{64}$ Immerhin wurde mit Verweis auf jüngere Bildungsromane bemerkt, dass die Geschichte der Gattung nicht abgeschlossen und ihre Funktion durchaus variabel sei. Dazu kam es jedoch nur am Rande, am Schluss eines Aufsatzes etwa. ${ }^{65}$ Im Mittelpunkt standen die Musterromane und ihre kanonischen Nachfolger, an denen sich eine dauerhaft gleichbleibende Funktion dieser Romanform aufzeigen ließ. Möglicherweise ist dies dem konservativen Zug eines teleologischen Funktionalismus geschuldet, der die soziale Integration oder Stabilität eines gesellschaftlichen Gesamtsystems als Bezugspunkt der Analyse voraussetzt. Der Funktionsbegriff jedenfalls ist für die Beschreibung historischen Wandels durchaus geeignet.

Zumindest die Literaturwissenschaft versteht unter »Funktion« nämlich die »p] lotentielle Wirkung eines Textes oder Textelements «. ${ }^{66}$ Damit steht zunächst eine »Disposition« oder »nachweisbare Eignung « in Rede. Der Begriff zielt »auf die potentielle Dienlichkeit einer Sache, nicht auf ihre tatsächliche Dienstbarkeit in einem einzelnen konkreten Fall «, ${ }^{67}$ mag die Letztere auch Gegenstand einer historischen Untersuchung sein, die sich für Textauslegungen und darüber hinaus für deren Konsequenzen interessieren muss, wenn denn gilt: »Unter Funktionen von Texten sollen jene Auswirkungen auf das Verhalten und Handeln ihrer Hörer/ Leser verstanden werden, die sich als Folgen der Textrezeption verstehen lassen. ${ }^{68}$ Wohl können Funktionen »nur über eine ästhetische Wirkungsstruktur realisiert

\footnotetext{
63 »Der Begriff >Institution< [...] soll das struktural-funktionale Moment des Romans und dessen deutlich abhebbares Organisationsprinzip bezeichnen. Dabei sind literarische Gattungen (wie der Roman) - auch darin Institutionen überhaupt vergleichbar - >keineswegs als statische Gegebenheiten zu betrachten; sie sind vielmehr einem fortlaufenden Prozeß der Institutionalisierung und Entinstitutionalisierung unterworfen «. Voßkamp (Anm. 6), 31.

64 Voßkamp (Anm. 5), 30.

65 Vgl. Voßkamp (Anm. 5), $37 \mathrm{f}$.

66 Harald Fricke, »Funktion«, in: Reallexikon der deutschen Literaturwissenschaft, Bd. 1, Berlin, New York 1997, 643-646, hier: 643 [Hv. MT].

67 Reinhold Schmücker, »Funktionen der Kunst«, in: Ders., Bernd Kleimann (Hrsg.), Wozu Kunst? Die Frage nach ihrer Funktion, Darmstadt 2001, 13-33, hier: 21.

68 Hans Ulrich Gumbrecht, »Poetizitätsdefinition zwischen Funktion und Struktur«, Poetica 10 (1978), 342-361, hier: 357.
} 
werden «. ${ }^{69}$ Die Analyse der Eigenstruktur eines Texts oder einer Gattung ist daher unerlässlich. Doch lässt sich ein Potenzial nicht als solches erschließen. Eine ästhetische Betrachtung allein reicht darum nicht hin; sie könnte über mögliche Wirkungen allenfalls spekulieren. Das Potenzial einer literarischen Gegebenheit entfaltet sich erst in der Geschichte seiner unterschiedlichen Realisierungen und kann nur an diesen studiert werden. So lassen sich anhand konkreter Einzeltexte und ihrer Rezeption exemplarisch Funktionsmöglichkeiten aufzeigen.

Wie bemerkt wurde, neigen funktionsgeschichtliche Ansätze allgemein dazu, eine bestimmte Realisierung des Potenzials dominant zu setzen und als kennzeichnend für den jeweiligen Gegenstand anzusehen. ${ }^{70}$ Auch die Behandlung des Bildungsromans weist diese Neigung auf. Die Bestimmung des Funktions- als eines Dispositionsbegriffs hält hingegen dazu an, Gattungen nicht auf die jeweils behandelte Funktion zu reduzieren, mag sie historisch auch vorherrschend sein. Gattungen erfüllen mitunter auch zur gleichen Zeit und im selben gesellschaftlichen Zusammenhang unterschiedliche Funktionen. Ein institutionengeschichtlicher Ansatz, der von der Überdeterminiertheit seines Gegenstands ausgeht, vermöchte einer solchen Multifunktionalität wohl Rechnung zu tragen. Ist er damit auch für die Untersuchung grenzüberschreitender Prozesse geeignet?

Dass diese Frage bislang kaum gestellt wurde, ist in dem methodologischen Nationalismus der Germanistik begründet wie auch in den Besonderheiten der deutschen Kolonialgeschichte und ihrer verspäteten Aufarbeitung. Während andere Philologien wie die Anglistik und die Romanistik, die auf eine sehr viel längere kolonialimperiale Vergangenheit zurückblickten, in der Folge der Dekolonisation von intellektuellen Repräsentanten der vormals Kolonisierten mit zunehmendem Nachdruck dazu angehalten wurden, Rechenschaft abzulegen, wirkten solche Impulse auf die Wissenschaft von der deutschen Literatur teils von außen, teils indirekt ein, vermittelt etwa durch die Globalgeschichte. Zweifellos gab der Postkolonialismus den stärksten Impuls zu einer transnationalen Wende. Aufgrund ihrer bevorzugten Behandlung der Literaturen Deutschlands und Frankreichs hatte die Komparatistik hierzulande die Naturalisierung der Nationalphilologien eher befördert. Wozu die Bestände der germanistischen Fachtradition unter den heutigen Bedingungen taugen, bleibt daher zu eruieren. Was die Funktionsgeschichte literarisch-sozialer Institutionen anbelangt, liegt es nahe, dabei die wissenschaftliche Vorgeschichte dieses Ansatzes einzubeziehen.

\section{III.}

Malinowski, der an der London School of Economics und später an der Yale University Anthropologie lehrte, hatte sich seinerzeit mit dem Kolonialismus und des-

\footnotetext{
69 Winfried Fluck, Das kulturelle Imaginäre. Eine Funktionsgeschichte des amerikanischen Romans 1790-1900, Frankfurt a. M. 1997, 19.

70 Marion Gymnich, Ansgar Nünning, »Funktionsgeschichtliche Ansätze: Terminologische Grundlagen und Funktionsbestimmungen von Literatur«, in: Dies. (Hrsg.), Funktionen von Literatur. Theoretische Grundlagen und Modellinterpretationen, Trier 2005, 3-27, hier: 11.
} 
sen Folgen zu befassen. Auf der Grundlage seiner funktionalistischen Kulturtheorie entwickelte er einen der ersten Versuche, Kulturkontakte systematisch in Betracht $\mathrm{zu}$ ziehen, was zu einer gewissen Dynamisierung seiner Konzeption führte. Das Hauptinteresse galt zu jener Zeit den Folgen der europäischen Kolonialherrschaft in Afrika.

Mit einem Begriff, der in den 1880er Jahren aufgekommen war, hatte man diese zunächst als acculturation erfasst. Malinowski, der zumeist von culture contact und culture change sprach, kannte bereits einen anderen Begriff, dem man heute, mit Blick auf die Verhältnisse des 21. Jahrhunderts, eine große Analysekraft beimisst: »> Transkulturalität<will, dem Doppelsinn des lateinischen trans- entsprechend, darauf hinweisen, dass [...] die kulturellen Determinanten heute quer durch die Kulturen hindurchgehen, sodass diese nicht mehr durch klare Abgrenzung, sondern durch Verflechtungen und Gemeinsamkeiten gekennzeichnet sind. ${ }^{71}$ Der Begriff ist unter Berufung auf Malinowski von dem kubanischen Anthropologen Fernando Ortiz geprägt worden. Er wollte damit die seinem Verständnis nach einseitige Anpassung meinende Rede von Akkulturation ersetzen. ${ }^{72}$ Malinowski verfasste für Contrapunteo cubano del tabaco y el azúcar (1940) ein Vorwort, das Transkulturation als eine wechselseitige Wirkung zwischen unterschiedlichen Kulturen auslegt, aus der etwas Neues hervorgeht. ${ }^{73}$ Mehr als punktuell verwandte er selbst diesen Begriff jedoch nur in dem letzten vor seinem Tode verfassten Aufsatz. ${ }^{74}$

Dieser letzte Aufsatz behandelt dasselbe Thema wie das 1949 posthum erschienene Buch The Dynamics of Culture Change. Es hat weniger einen symmetrischen Austausch zum Gegenstand als vielmehr die einseitige Auswirkung der Kolonisierung auf die Gesellschaften Afrikas. Das Interesse an kulturellem Wandel entsprach einer allgemeinen Tendenz der Anthropologie, die Mitte des 20. Jahrhunderts auf den Untergang der meisten »primitiven Gesellschaften « - und somit auf den Verlust ihres angestammten Forschungsobjekts - mit einer Hinwendung zu »Übergangsge-

\footnotetext{
71 Wolfgang Welsch, »Was ist eigentlich Transkulturalität?«, in: Lucyna Darowska, Thomas Lüttenberg, Claudia Machold (Hrsg.), Hochschule als transkultureller Raum? Kultur, Bildung und Differenz in der Universität, Bielefeld 2010, 39-66, hier: 42 (Hv. i. O.).

72 »Entendemos que el vocablo transculturación expresa mejor las diferentes fases del proceso transitivo de una cultura a otra, porque éste no consiste solamente en adquirir una distinta cultura, que es lo que en rigor indica la voz anglo-americana aculturation, sino que el proceso implica también necesariamente la pérdida o desarraigo de una cultura precedente, lo que pudiera decirse una parcial desculturación, y, además, significa la consiguiente creación de nuevos fenómenos culturales que pudieran denominarse de neoculturación. Al fin, como bien sostiene la escuela de Malinowski, en todo abrazo de culturas sucede lo que en la cópula genética de los individuos: la criatura siempre tiene algo de ambos progenitores, pero también siempre es distinta de cada uno de los dos. En conjunto, el proceso es una transculturación, y este voca-blo comprende todas las fases de su parábola. «Fernando Ortiz, Contrapunteo cubano del tabaco y el azúcar [1940], Caracas 1978, $96 \mathrm{f}$.

73 »Es un proceso en el cual ambas partes de la ecuación resultan modificadas. Un proceso en el cual emerge una nueva realidad, compuesta y compleja; una realidad que no es una aglomeración mecánica de caracteres, ni siquiera un mosaico, sino un fenómeno nuevo, original e independiente.« Bronisław Malinowski, »Introduccion«, in: Ortiz (Anm. 72), 3-10, hier: 5.
}

74 Malinowski (Anm. 38), 650. 
sellschaften « im Prozess der bald so genannten »Modernisierung « reagierte. ${ }^{75}$ Die Wissenschaft wusste um ihre aktive Teilhabe an diesem Prozess. Eine »practical anthropology $\ll^{76}$ wollte »for the task of assisting colonial control «77 nützlich sein. Daraus, dass er seine funktionalistische Theorie des Kulturkontakts mit der Perspektive und im Dienst kolonialer Herrschaft, und zwar zum Zweck ihrer Optimierung im Rahmen eines »social engineering ${ }^{78}{ }^{78}$ entwickelte, machte Malinowski keinen Hehl: »The practical value of such a theory is that it teaches us the relative importance of various customs, how they dovetail into each other, how they have to be handled by missionaries, colonial authorities, and those who economically have to exploit savage trade and savage labour. $\ll^{79}$

Dessen ungeachtet ist der funktionalistische Grundsatz bedenkenswert, dass kultureller Wandel nicht an einzelnen, isolierbaren culture traits und ihrer Diffusion $\mathrm{zu}$ studieren ist, sondern nur ganzheitlich mit Blick auf Interdependenzen, und zwar auf der Ebene des Institutionellen erfasst werden kann. ${ }^{80}$ Diese Auffassung erweist sich hinsichtlich der seit den 1970er Jahren so genannten »Globalisierung « als aktuell. Nach Giddens ist dieser Prozess nicht anders denn als »Ausbreitung der modernen Institutionen über die ganze Welt $\ll^{81} \mathrm{zu}$ betrachten, wobei auf »Institutionenbündelungen $\ll^{82} \mathrm{zu}$ achten sei. John W. Meyer spricht in dieser Hinsicht von einer »Weltkultur«. Da der von ihm begründete Neoinstitutionalismus im Folgenden für eine Reformulierung des literaturwissenschaftlichen Ansatzes im Lichte gegenwärtiger Probleme herangezogen werden soll, sei dessen Institutionenbegriff kurz erläutert: Er zielt auf »kulturelle Regeln, die bestimmten Einheiten und Handlungen kollektiven Sinn und Wert verleihen und sie in einen größeren Rahmen integrieren $\ll .{ }^{83}$ Es sind vor allem drei Einheiten oder $»$ Akteure $\ll$, die der Neoinstitutionalismus als die wichtigsten Strukturformen der modernen Gesellschaft be-

\footnotetext{
75 So galt es für Malinowski zunächst, einer »antiquarian and romantic tendency toward the retrospective and reconstructive« zu begegnen: »[T]he scientific anthropologist must be the anthropologist of the changing Native. Why? Because what exists nowadays is not a primitive culture in isolation but one in contact and process of change. « Bronisław Malinowski, The Dynamics of Culture Change. An Inquiry into Race Relations in Africa, New Haven 1949, 6.

76 Bronisław Malinowski, »The rationalization of anthropology and administration«, Africa 3/4 (1930), 405-430, hier: 408-411.

77 Malinowski (Anm. 76), 408.

78 Malinowski (Anm. 75), 6.

79 Bronisław Malinowski, »The Life of Culture«, in: Ders. u. a. (Hrsg.), Culture: The Diffusion Controversy, New York 1927, 23-42, hier: 36.

80 Vgl. Malinowski (Anm. 75), 19-22.

81 Anthony Giddens, Konsequenzen der Moderne, Frankfurt a. M. 1995, 83.

82 Giddens (Anm. 81), 84.

83 John W. Meyer, John Boli, George M. Thomas, »Ontologie und Rationalisierung im Zurechnungssystem der westlichen Kultur«, in: John W. Meyer, Weltkultur. Wie die westlichen Prinzipien die Welt durchdringen, Frankfurt a. M. 2005, 17-46, hier: 18. Institutionen sind also von Organisationen zu unterscheiden. Schon Schelsky hatte die Institution als »ein soziales Gebilde« verstanden, »dessen Dauerhaftigkeit und Stabilität tiefer begründet ist als auf dem planenden Zweckhandeln und dessen Funktion für das Leben der Gesamtgesellschaft fundamentaler und unersetzbarer ist als die von der Veränderung bewußter Ziele und Interessen her schneller wechselnden Organisationen und Organisationsformen. " Helmut Schelsky, »Ist die Dauerreflexion institutionalisierbar? Zum Thema einer modernen Religionssoziologie « [1957], in: Ders., Auf der Suche nach der Wirklichkeit (Anm. 29), 250-275, hier: 262.
} 
handelt: die formale Organisation, der souveräne Nationalstaat und das autonome Individuum. ${ }^{84}$

Meyers Konzeption der Institution als basale gesellschaftliche Erwartungsstruktur ist an Max Webers Theorie der Rationalisierung angelehnt ${ }^{85}$ und im Sinne von Berger und Luckmann wissenssoziologisch fundiert. ${ }^{86}$ Sie betont mithin ein kognitives Moment, zielt nämlich auf Modelle und »Skripts«, die Handlungsprobleme definieren und Handelnde mit Motiven versehen. In Prozessen der Institutionalisierung werden demnach sowohl Handlungsmuster konstituiert als auch allererst, darauf legt Meyer großes Gewicht, die Akteure. Das gilt für die drei genannten Formen von Akteurschaft gleichermaßen. Im Zusammenhang mit dem Bildungsroman ist Individualität eine besondere Relevanz einzuräumen, wenngleich die Bündelung mit anderen Formen untersucht werden muss. Zu neueren germanistischen Arbeiten über die »Erfindung « des Individuums im 18. Jahrhundert passt Meyers diesbezügliche These: »Das Individuum ist ein institutioneller Mythos, der aus rationalisierten Theorien ökonomischen, politischen und kulturellen Handelns entsteht «. ${ }^{87}$

Die Erzählungen der Literaten, so wäre zu ergänzen, haben den »institutionalisierten Individualismus $\aleph^{88}$ mitbegründet. Talcott Parsons, der diese Formel mit Blick auf die amerikanische Gesellschaft der Zeit nach dem Zweiten Weltkrieg geprägt hat, verband Webers Theorie des sozialen Handelns mit Durkheims Konzeption der sozialen Tatsachen zu der Annahme, dass sich Akteure an einem kollektiven Werte- und Normensystem orientieren, das im Zuge der Sozialisation internalisiert wird. Individualität zählt zu den zentralen Werten der modernen Gesellschaft. Schon Durkheim stellte zu seiner Zeit fest, der Individualismus stelle »fortan das einzige Glaubenssystem dar, das die moralische Einheit eines Landes sicherstellen kann « ${ }^{89}$ Seine Rede vom »Kult des Individuums « hebt hervor, dass dieser in der säkularisierten, arbeitsteiligen Gesellschaft die religiöse Moral ablöst: Das Individuum nimmt den Platz Gottes ein.

Neben der religionssoziologischen findet sich bei Durkheim auch die strukturtheoretische These, Individualisierung sei eine Begleiterscheinung gesellschaftlicher Differenzierung. Ähnlich ordnet Niklas Luhmann, dem die jüngere Germanistik folgt, ${ }^{90}$ die »Semantik« der Individualität ein. Demgegenüber versteht Meyer institutionali-

\footnotetext{
84 Siehe dazu John W. Meyer, Roland Jepperson, »The >Actors< of Modern Society: The Cultural Constitution of Social Agency«, Sociological Theory 18 (2000), 100-120.

85 Siehe dazu John W. Meyer, Gili S. Drori, Hokyu Hwang (Hrsg.), »World Society and the Proliferation of Formal Organization«, in: Dies. (Hrsg.), Globalization and Organization. World Society and Organizational Change, Oxford 2006, 25-49, hier: 27-29.

86 Sieh dazu Renate E. Meyer, »New Sociology of Knowledge. Historical Legacy and Contributions to Current debates in Institutional Research«, in: Royston Greenwood u. a. (Hrsg.), The SAGE Handbook of Organizational Institutionalism, Los Angeles 2008, 519-538.

87 Meyer, Boli, Thomas (Anm. 83), 35.

88 Talcott Parsons, Winston White, »The Link Between Character and Society«, in: Talcott Parsons, Social Structure and Personality, New York 1964, 183-235, hier: 197.

89 Émile Durkheim, »Der Individualismus und die Intellektuellen« [1889], in: Hans Bertram (Hrsg.), Gesellschaftlicher Zwang und moralische Autonomie, Frankfurt a. M. 1986, 54-70, hier: 62.

90 Siehe etwa Fotis Jannidis, Das Individuum und sein Jahrhundert. Eine Komponenten- und Funktionsanalyse des Begriffs >Bildung am Beispiel von Goethes »Dichtung und Wahrheit«, Tübingen 1996.
} 
sierten Individualismus in dem bereits angesprochenen Sinne als ein Kulturmuster, das sich nicht derivativ zu vermeintlich tiefer liegenden Sozialstrukturen verhält. Es ist deshalb, darauf kommt es hier an, hoch beweglich und kann als eigenständiger Faktor auch dort wirksam werden, wo die angeblich erforderlichen Strukturbedingungen wie in manchen »Entwicklungsländern« der »Dritten Welt « nicht aufweisbar sind. So ist der Befund einer erstaunlichen Gleichförmigkeit des Handelns erklärlich, auf dem die Theorie der Weltkultur fußt. Und zwar zieht sie zur Erklärung »institutioneller Isomorphie« drei unterschiedliche Mechanismen in Betracht: Nachahmung, normativer Druck und Zwang. ${ }^{91}$

Der Hinweis auf kulturelle Prozesse der Diffusion stellt für funktionalistische Ansätze zunächst deshalb eine Herausforderung dar, weil er eine Rückführung von Institutionen auf Bedürfnisse problematisch erscheinen lässt - zumal dann, wenn kulturelle Muster oktroyiert werden. Jene kolonialzeitlichen Institutionentransfers, die Malinowski vor Augen hatte, beruhten auf asymmetrischen Machtverhältnissen. Seine besondere Aufmerksamkeit zog unter dem Aspekt des Kulturkontakts das koloniale Erziehungswesen auf sich: »Education, under normal conditions, is the transmission of culture from one generation to another. Under conditions of culture change or transculturation it implies not merely the transmission of one system but the welding-together of two. ${ }^{92}$ Literaturgeschichtlich ist diese Beobachtung insofern von besonderem Interesse, als man sich literarischer Texte im Rahmen des kolonialen Schulsystems zum Zweck der Verbreitung europäischer Normen und Werte bediente. Mit Blick auf Indien hat Gauri Viswanathan »literary education, as opposed to literature, as a major institutional support system of colonial administration « bezeichnet und auf die Funktion humanistischer Bildung in diesem Zusammenhang hingewiesen: »[C]ertain humanistic functions traditionally associated with literature - for example, the shaping of character or the development of the aesthetic sense or the disciplines of ethical thinking - were considered essential to the processes of sociopolitical control by the guardians of the same tradition. $\ll^{93} \mathrm{Zu}$ den literarischen Werken, die auf diese Weise in die britischen Kolonien gelangten, zählten auch Bildungsromane. Eine transkulturelle Funktionsgeschichte dieser Gattung hat mithin dem Umstand Rechnung zu tragen, dass ihre Institutionalisierung in manchen Gesellschaften erzwungen wurde - eine Rückführung auf Bedürfnisse der Leserschaft, die wohl an der Idee eines freien Markts orientiert ist, erweist sich in solchen Fällen als besonders fragwürdig.

Überhaupt ist festzustellen, dass der frühen Rede von »Transkulturation« ein $\mathrm{Pa}$ thos der Grenzüberwindung und damit der euphorisch hoch gestimmte Ton, in dem sie heute vielfach vorgetragen wird, gänzlich fremd ist. Malinowski gelangte zu seiner Einschätzung der transkulturellen Situation afrikanischer Gesellschaften als »both complex and fraught with dangerous (not to say tragic possibilities) « ${ }^{94}$ unter

\footnotetext{
91 Paul J. DiMaggio, Walter W. Powell, »The Iron Cage Revisited. Institutional Isomorphism and Collective Rationality in Organizational Fields «, American Sociological Review 48 (1983), 147-160.

92 Malinowski (Anm. 38), 652.

93 Gauri Viswanathan, Masks of Conquest: Literary Study and British Rule in India, New York 1989, 4 (Hv. i. O.).

94 Malinowski (Anm. 38), 652.
} 
der Voraussetzung, dass es sich dabei um organische Einheiten handelt, die sich in einem Gleichgewichtszustand befinden oder nach einer Störung dessen Wiederherstellung anstreben. Dementsprechend betont er »forces of conservatism inherent in Native institutions «. ${ }^{95}$ Eben die Stärke dieses Ansatzes: dass er Interdependenzen zwischen unterschiedlichen Institutionen erfasst, erweist sich als Schwäche, wo die innergesellschaftlichen Bezüge, der Organismus-Analogie folgend, überbetont werden.

Mit Blick auf die frühe Anthropologie »primitiver « Kulturen mag man dieser Betrachtungsweise eine gewisse Berechtigung zubilligen, insofern die Feldforschung anfangs vornehmlich kleine Gruppen in den Blick nahm, die teils weitgehend isoliert waren. Dass Kulturen wie jene der Trobriander im frühen 20. Jahrhundert vergleichsweise wenig dynamisch und somit der synchronen Analyse besser zugänglich waren als die Kulturen des unter europäischer Einwirkung stehenden Afrika etwa, ist gut vorstellbar. Im Falle der Geschichts-, der Sozial- und auch der Literaturwissenschaften, die sich mit »modernen « Gesellschaften befassen, zollt die Beschränkung der Perspektive auf den Raum eines Territorialstaats dagegen der Wertvorstellung nationaler Einheit Tribut. In beiden Zusammenhängen erwies sich, auch bedingt durch einen Wertewandel, die Voraussetzung räumlich begrenzter Untersuchungseinheiten zunehmend als problematisch, wenn nicht haltlos.

Daher rührt die jüngere Konjunktur von »Transkulturation« und anderen »Trans«Begriffen. Programmatisch bekunden sie einen Willen zur analytischen Grenzüberwindung, der vielfach auch die Wahrnehmung entfernter Zeiten verändert. Die starke These, Transkulturation sei immer schon der Fall, bringt jede historische Darstellung unterschiedlicher Kulturen in Misskredit. Damit wird der Funktionsbegriff eigentlich unbrauchbar, setzt er doch den Bezugsrahmen einer begrenzten Einheit voraus. Es scheint indes ratsam, die traditionelle Vorstellung einer Mehrzahl unterschiedlicher Kulturen nicht pauschal zu verwerfen, sondern einem neueren Denkbild folgend zu revidieren. In der Auseinandersetzung mit der organologischen Tradition ist ein Konzept der »Assemblage « entwickelt worden, das dazu anleitet, dynamische Ensembles aus heterogenen Elementen zu beschreiben. ${ }^{96}$ Der $»$ nouveau fonctionalisme $\ll{ }^{97} \mathrm{Mi}$ chel Foucaults verwendet dazu den ähnlichen Begriff »dispositif«, wobei auch er davon ausgeht, ein solches Ensemble habe »pour fonction majeure de répondre à une urgence $\ll{ }^{98}$ Dass es mobile Elemente in sich aufnimmt oder aus sich entlässt, hindert nicht daran, es als temporär stabile Ganzheit aufzufassen. Begreift man Kulturen als Assemblagen, kann durchaus nach der Funktion ihrer zeitweiligen Bestandteile gefragt werden.

\footnotetext{
95 »A comprehensive institution endures because it is organically connected and satisfies an essential need of society. It can be suppressed, but is then driven underground. It can be mutilated, deprived of this or that aspect or prerogative, but it disappears only with the destruction of the whole cultural identity of a people.« Malinowski (Anm. 75), 53.

96 Siehe dazu Marcus Twellmann, »Assemblage (Collage, Montage): für einen neuen Formalismus«, DVjs 93 (2019), 239-261.

97 Gilles Deleuze, Michel Foucault, Paris 2004, 33.

98 Michel Foucault, »Le Jeu de Michel Foucault«, in: Ders., Dits et écrits, Bd. 3: 1976-1979, Paris 1994, 298-329, hier: 299.
} 
Der Befund einer historischen Variabilität lässt vermuten, dass Funktionen auch kulturell einer Veränderung fähig sind. Wo Institutionen sich räumlich verbreiten und über kulturelle Grenzen hinweggelangen, könnten sie sich mithin als geeignet für Zwecke erweisen, die man ihnen im Zusammenhang ihrer Entstehung nicht zugedacht hatte. Aus diesem Grund erkennt Andreas Reckwitz dem Konzept der nicht antizipierten Konsequenzen, das von Giddens der funktionalistischen Erbmasse entnommen wurde, eine besondere Aktualität zu: »Es sind die Beziehungen zwischen sozialen Praktiken an differenten Orten bzw. zu differenten historischen Zeitpunkten, denen das Interesse gilt und die unter dem Gesichtspunkt > unintendierter Folgen < zu untersuchen sind. «99 Im Unterschied zum herkömmlichen, teleologischen Funktionalismus habe ein kontingenztheoretischer Funktionalismus die Relation zwischen Praktiken unterschiedlicher Räume und Zeiten nicht als eine Sequenz von Ursache und Wirkung, sondern als nichtdeterminierten Prozess in den Blick zu nehmen. Im vorliegenden Zusammenhang ist die Beobachtung interessant, dass in vielen Analysen aus dem Umfeld der postkolonialen Theorie zumindest implizit unintendierten Handlungsfolgen ein prominenter Stellenwert zukommt. ${ }^{100}$ Gemeint sind solche Analysen, die von der kreativen Aneignung kultureller, nicht zuletzt literarischer Praktiken handeln.

Es wurde eingangs bereits darauf hingewiesen: Selbst eine so spezifisch deutsche Gattung wie der Bildungsroman hat sich als grenzüberschreitend beweglich erwiesen, und zwar auch außerhalb von Europa. Nicht zuletzt fand sie den Weg in kolonisierte Länder, wo sie auch nach der Dekolonisation weiter Verbreitung fand. Bei der Untersuchung kultureller Mobilität, das wusste bereits Malinowski, ist stets von einer Veränderung auszugehen: »Just because no idea and no object can exist in isolation from its cultural context, it is impossible to sever mechanically an item from one culture and place it in another. The process is always one of adaptation in which the receiving culture has to re-evolve the idea, custom, or institution which it adopts. « ${ }^{101}$ Dass dies auch für literarische Gattungen gilt, demonstrieren die Forschungsbeiträge zum Thema des postkolonialen Bildungsromans: Sie handeln von vielfältigen Adaptionen.

Die Rede von »generic transculturation « taucht in diesem Zusammenhang kaum überraschend auf. Sie ist zugleich beispielhaft für die lange Dauer funktionalistischer Denkgewohnheiten. Maria Helena Lima übernimmt den Transkulturationsbegriff nicht direkt von Ortiz, sondern vermittelt durch Ángel Rama, der ihn für eine Untersuchung lateinamerikanischer Erzählliteratur nutzbar gemacht hatte. Zuerst 1974 im Rahmen eines Aufsatzes und 1982 in Buchform hatte Rama Studien zur Entstehung eines literarischen Regionalismus vorgelegt, den er in Zusammenhang mit Prozessen der Modernisierung sieht, die von den Städten ausgingen. Er versieht Ortiz' Begriff mit einem Akzent auf der »Energie« und »Kreativität« lebendiger kul-

\footnotetext{
99 Andreas Reckwitz, »Der verschobene Problemzusammenhang des Funktionalismus: Von der Ontologie der sozialen Zweckhaftigkeit zu den Raum-Zeit-Distanzierungen«, in: Jens Jetzkowitz, Carsten Stark (Hrsg.), Soziologischer Funktionalismus. Zur Methodologie einer Theorietradition, Opladen 2003, 57-81, hier: 74.

100 Reckwitz (Anm. 99), 71.

101 Malinowski (Anm. 79), 27.
} 
tureller Gemeinschaften des Hinterlands. ${ }^{102}$ Während Rama neben Ortiz auch andere Anthropologen heranzieht, nimmt Limas Aufsatz aus dem Jahr 1993 auf diese Tradition nicht mehr Bezug. Gleichwohl liegen funktionalistische Annahmen auch ihrer Untersuchung zugrunde: »[D]ifferent cultures will transform the >originary< genre to serve their particular needs ${ }^{103}$ - offenbar kommen auch Transkulturationsstudien nicht gut ohne die Annahme aus, es gebe unterscheidbare Kulturen, denen sich besondere Bedürfnisse zuschreiben lassen, in deren Erfüllung ein Zweck erkannt werden kann.

\section{IV.}

Die in postkolonialen Studien vielfach verfolgte Frage nach »nicht antizipierten Konsequenzen « hatte Robert K. Merton in einem früheren Aufsatz zunächst mit Blick auf einzelne Handlungen entfaltet, ${ }^{104}$ bevor er sich Ende der 1940er Jahre institutionellen Handlungsmustern zuwandte. Unter dem Titel »Manifest and Latent Functions « hat er drei zentrale Postulate des Funktionalismus revidiert und den analytischen Rahmen derart erweitert, dass nun auch gesellschaftlichen Konflikten in einem gewissen Umfang Rechnung getragen werde konnte. So wird das Postulat, sämtliche Formen einer Gesellschaft hätten positive, insbesondere bestandserhaltende Funktionen, mit dem Hinweis zurückgewiesen, dass sie mitunter aus Sicht von »subgroups sufficiently powerful to retain these forms intact, by means of direct coercion or indirect persuasion $«,{ }^{105}$ wohl funktional, aus Sicht anderer Teilgruppen dagegen dysfunktional sein können. Unter dieser Voraussetzung ließen sich auch asymmetrische Machtbeziehungen berücksichtigen, wie sie in kolonialgeschichtlichen Zusammenhängen bestanden und auch seither fortbestehen.

Der wohl bedeutendste Diskussionsbeitrag ist die im Titel des Aufsatzes annoncierte Distinktion. Diese hebt nicht auf unterschiedliche Teilnehmerperspektiven ab, sondern setzt die Möglichkeit einer Außenperspektive voraus, wie sie im Fall der ethnologischen Untersuchung einer fremden Kultur gegeben $\mathrm{zu}$ sein scheint. Als »latent « werden solche Funktionen bezeichnet, die von den Absichten der Akteure abweichen und von diesen auch nicht erkannt werden. ${ }^{106}$ Das wird exemplarisch an den Regentänzen der Hopi-Indianer verdeutlicht: Diese führten zwar nicht zum erklärten Ziel, festigten jedoch den Gruppenzusammenhalt. Es bedürfe der Perspektive eines externen Beobachters, eines Soziologen, um dies zu entdecken. Merton hält die Erkennung solcher Latenzen für die eigentliche Aufgabe der Soziologie

\footnotetext{
102 Vgl. Ángel Rama, Transculturación narrativa en América Latina [1982], Buenos Aires 2007, 45, 41.

103 Maria Helena Lima, »Decolonizing Genre: Jamaica Kincaid and the Bildungsroman«, Genre 26 (1993), 431-459, hier: 433.

104 Robert K. Merton, »The Unanticipated Consequences of Purposive Social Action«, American Sociological Review 1/6 (1936), 894-904.

105 Robert K. Merton, »Manifest and Latent Functions«, in: Ders., Social Theory and Social Structure, New York 1968, 73-138, hier: 86.
}

106 Vgl. Merton (Anm. 105), 105. 
und ihren gesellschaftlichen Beitrag - die manifesten Funktionen seien ja ohnehin bekannt. ${ }^{107}$

Eine der Redeformen, in denen sie bekanntgemacht werden, sind jene Selbstverständniserklärungen, die vom Zweck einer Institution handeln und so legitimierend wirken sollen. Solche hatte auch Malinowski bereits im Blick. Er bezeichnete »the system of values for the pursuit of which human beings organize, or enter organizations already existing ${ }^{108}$ als charter. Schelsky rückt diesen neben Maurice Harious Begriff der idée directrice. ${ }^{109}$ Gehlen hatte mit »Führungsnorm « übersetzt. ${ }^{110} \mathrm{Ge}$ meint sind grundlegende und leitende Ideen, die Zustimmung herbeiführen.

Malinowski unterstreicht, dass es dabei auf die Perspektive der Akteure ankommt, wenn er von »the recognized purpose of the group« und »the idea of the institution as entertained by its members and defined by the community « ${ }^{111}$ spricht. Eben deshalb aber gilt es, die Charter einer Institution von ihrer Funktion zu unterscheiden. Damit ist einerseits die Konzeption einer nicht erkannten, latenten Funktion vorbereitet, anderseits aber auch die Funktionalismuskritik einer Wissenssoziologie, die eine konsequente Übernahme der Akteursperspektive einfordert - jenes »native's point of view «112 gewissermaßen, dessen Gewinnung Malinowski selbst einmal zur Maxime der Ethnografie erklärt hatte. Gegen die Funktionszuschreibung aus externer Beobachterperspektive erheben Peter L. Berger und Thomas Luckmann nämlich den Einwand, »daß >Funktionen< wie >Dys-Funktionen< nur auf der Ebene von Sinn analysiert werden können. Demzufolge bedeutet >funktionale Integration $<-$ will man den Ausdruck überhaupt verwenden - die Integration einer institutionalen Ordnung mithilfe ihrer Legitimationsprozesse. «113

Diese Unterscheidung könnte für Zwecke der Literaturgeschichtsschreibung nützlich sein. Wer nach dem historischen Sinn des Bildungsromans fragt und bei seiner Charter ansetzt, der wird sich etwa für die erklärten Wirkungsabsichten von Autoren oder für poetologische Zwecksetzungen von Seiten der Kritiker und Philologen interessieren. Mitunter sprechen auch fiktive Romanfiguren die leitende Idee aus, so Goethes Wilhelm: »mich selbst, ganz wie ich da bin, auszubilden «. ${ }^{114}$ Christian Gottfried Körner teilte im Jahr der Romanpublikation in einem Brief an Friedrich Schiller seine Idealvorstellung mit: »Das Ziel dieser Ausbildung ist ein

\footnotetext{
107 Vgl. Merton (Anm. 105), 120.

108 Malinowski (Anm. 34), 52.

109 Schelsky (Anm. 13), 16.

110 Vgl. Gehlen (Anm. 24), 479.

111 Malinowski (Anm. 34), 48.

112 Bronisław Malinowski, Argonauts of the Western Pacific. An account of native enterprise and adventure in the Archipelagoes of Melanesian New Guinea [1922], New York 1961, 25.

113 Peter L. Berger, Thomas Luckmann, Die gesellschaftliche Konstruktion der Wirklichkeit: Eine Theorie der Wissenssoziologie, Frankfurt a. M. 1980, 87.

114 Johann Wolfgang Goethe, Wilhelm Meisters Lehrjahre. Ein Roman, in: Ders., Sämtliche Werke nach Epochen seines Schaffens. Münchner Ausgabe, hrsg. Karl Richter u.a. München, Wien 1988, Bd. 5., hrsg. Hans-Jürgen Schings, Karl Richter, München, Wien 1988, 288.
} 
vollendetes Gleichgewicht - Harmonie mit Freiheit. «115 Daniel Jenisch wies im Folgejahr auf die gesellschaftlich befriedende Wirkung des Romans hin: »Meisters Lehrjahre machen uns zufrieden mit einem eingeschränkten Lebens-Kreise, machen uns jede Pflicht menschlichen Verhältnisses nur desto heiliger, desto liebens- und achtungswerther. ${ }^{116}$ Auch Karl Morgenstern ist hier anzuführen, der den Begriff als erster verwandte und sich in Vorträgen der Jahre 1819 und 1820 ausführlicher zur Funktionsfrage äußerte: Mit dem »bisher nicht üblichen Worte [...] Bildungsroman ${ }^{117}$ bezeichnet er einen solchen Roman, der Bildung erstens »darstellt« und zweitens »fördert «. ${ }^{118}$ Mithin gibt der Name der Gattung nicht nur ihren Gegenstand an, sondern auch ihren Zweck: Der Bildunngsroman ist ein Mittel zur Bildung.

Wenn sie von einer harmonisierenden Vorstellung auch abrückte, so hielt die Poetologie an dieser instrumentellen Zuschreibung doch bis in die jüngste Vergangenheit fest. Die Deutung der Gattung als »Handlungsanleitung und Verstehenshilfe bürgerlicher Existenz «, ${ }^{119}$ vermag auch deshalb zu überzeugen, weil sie einer allgemeineren Bestimmung entspricht: »[L]a littérature «, so äußerte sich Roland Barthes gleichfalls im funktionalistischen Duktus jener Zeit, »est cet ensemble d'objets et de règles, de techniques et d'œuvres, dont la fonction dans l'économie générale de notre société est précisément d'institutionnaliser la subjectivité. «120 Die Funktionsgeschichte des Bildungsromans scheint eben dies für das bürgerliche Zeitalter zu zeigen: Die Literatur vermittelt ihrem Leser ein Selbstverständnis als Individuum und dazugehörige Techniken der praktischen Selbstführung. Zweifellos zählt Bildung seit mehr als zwei Jahrhunderten zu den »obersten Führungssystemen «121 einer abnehmend religiös orientierten Gesellschaft. Und der kunstvolle Umgang mit Schrift ist in diesen Zusammenhang stark eingebunden. Mit einem geweiteten Blick auf den europäischen Bildungsroman und die gesellschaftliche Moderne erklärt auch der Komparatist Franco Moretti, die literarische Darstellung von Sozialisation trage einen Widerspruch zwischen den Anforderungen der Gesellschaft und dem Ideal der individuellen Selbstbestimmung aus und lehre den Leser, damit zu leben. ${ }^{122}$ Ähnlich versteht Voßkamp diesen Romantyp als »Ausdruck der Moderne und Antwort auf

\footnotetext{
115 [Christian Gottfried Körner], »Über Wilhelm Meisters Lehrjahre (aus einem Brief an den Herausgeber der Horen)«, in: Klaus F. Gille (Hrsg.), Goethes Wilhelm Meister. Zur Rezeptionsgeschichte der Lehr- und Wanderjahre, Königstein/Ts. 1979, 8-14, hier: 10 (Hv. i. O.).

116 Daniel Jenisch, Ueber die hervorstechendsten Eigenthümlichkeiten von Meisters Lehrjahren oder ueber das, wodurch dieser Roman ein Werk von Goethen's Hand ist. Ein ästhetisch-moralischer Versuch, Berlin 1797, 19.

117 Karl Morgenstern »Über das Wesen des Bildungsromans« [1820], in: Rolf Selbmann (Hrsg.), Zur Geschichte des Bildungsromans, Darmstadt 1988, 55-72, hier: 55.

118 Karl Morgenstern, »Zur Geschichte des Bildungsromans« [1824], in: Selbmann (Hrsg.) (Anm. 117), 73-99, hier: 74.

119 Voßkamp (Anm. 61), 342.

120 Roland Barthes, »Histoire et littérature: à propos de Racine«, Annales 15/3 (1960), 524-537, hier: 537.

121 Gehlen (Anm. 24), 453.

122 Vgl. Moretti (Anm. 54), 10.
} 
die Moderne «, ${ }^{123}$ wobei der Germanist allerdings die »eigentümlichen Dispositionen und Bedürfnisse des Bildungsbürgertums in Deutschland « ${ }^{124}$ betont.

Bedenkt man den Unterschied von Charter und Funktion, dann scheint allerdings fraglich, ob Verlautbarungen wie jene der von Voßkamp in dieser Sache als Zeugen beigebrachten Körner, Jenisch und Morgenstern tatsächlich dazu geeignet sind, die gesellschaftliche Funktion des Bildungsromans zu erschließen. Wäre die frühe Romantheorie nicht besser als Rechtfertigungsmythos der entstehenden Institution zu behandeln? Morgenstern, von Beruf Professor für Eloquenz und Klassische Philologie, Ästhetik und Geschichte der Literatur und Kunst, teilte seine diesbezüglichen Überlegungen im Zusammenhang einer Reihe von Reden und Aufsätzen mit, die das universitäre Programm eines Studium generale empfehlen. Darin bezieht er, so Fritz Martini, das auf die antike Rhetorik zurückgehende Erziehungsideal einer allseitigen Entfaltung menschlicher Fähigkeiten auf die neue Romanform. ${ }^{125}$ Ersichtlich dient das Programm nicht allein deren Rechtfertigung. Auch die Philologie soll damit legitimiert werden. Besonders der Neuphilologie musste daran gelegen sein, ihre gesellschaftliche Funktion auszuweisen. Generell ist der Aufstieg des Romans zur vorherrschenden Literaturform des 19. Jahrhunderts von der Etablierung dieser anderen Institution nicht zu trennen. ${ }^{126}$ Im Verbund mit dem Bildungsroman arbeitete die universitäre »Literaturwissenschaft«, wie sie später heißen sollte, an der Institutionalisierung von Subjektivität im Modus der Individualisierung.

Am Ende des Jahrhunderts gab Wilhelm Dilthey, der lange Zeit als Erfinder des Begriffs galt, dem Mythos dann seine wohl wirksamste Formulierung. ${ }^{127}$ Wer sich an solchen Deklarationen orientiert, der wird kaum einen Text finden, der ihnen entspricht. Er wird die wissenschaftliche Rede vom Bildungsroman zu den »many legends in literary history « zählen. ${ }^{128}$ Wenn auch unbeabsichtigt, so doch treffend ist mit diesen Worten die institutionelle Funktion idealisierender Poetik bezeichnet. Ihre Betrachtung als Charter könnte eine in variierender Begrifflichkeit vielfach konstatierte Divergenz erhellen - zu unintendierten Folgen kam es schon vor jeder Transkulturation!

\footnotetext{
123 Voßkamp (Anm. 61), 348.

124 Voßkamp (Anm. 61), 348.

125 Vgl. Fritz Martini, »Der Bildungsroman. Zur Geschichte des Wortes und der Theorie«, DVjs 35 (1961), 44-63, hier: $48 \mathrm{f}$.

126 Siehe für England und Nordamerika Homer Brown, »Prologue: why the story of the origin of the (English) novel is an American romance (if not the great American novel)«, in: Deirdre Lynch, William Warner (Hrsg.), Cultural Institutions of the Novel, Durham 1996, 11-43.

127 »Eine gesetzmäßige Entwickelung wird im Leben des Individuums angeschaut, jede ihrer Stufen hat einen Eigenwert und ist zugleich Grundlage einer höheren Stufe. Die Dissonanzen und Konflikte des Lebens erscheinen als die notwendigen Durchgangspunkte des Individuums auf seiner Bahn zur Reife und zur Harmonie. Und >höchstes Glück der Erdenkinder< ist die >Persönlichkeit<, als einheitliche und feste Form des menschlichen Daseins. Nie ist dieser Optimismus der persönlichen Entwickelung, der auch Lessings harten Lebensweg erleuchtet hat, heiterer und lebenssicherer ausgesprochen worden als in Goethes Wilhelm Meister: ein unvergänglicher Glanz von Lebensfreude liegt auf diesem Romane und denen der Romantiker.« Wilhelm Dilthey, Das Erlebnis und die Dichtung, in: Ders., Gesammelte Schriften, Bd. 26, Göttingen 2005, 253.
}

128 Jeffrey L. Sammons, »The Mystery of the Missing Bildungsroman, or: What Happened to Wilhelm Meisters Legacy?«, Genre 14 (1981), 229-246, hier: 243. 
Voßkamp unterscheidet zwischen »Rezeptionsgeschichte (als Auslegungs- und Konzeptionsgeschichte) ${ }^{129}$ und $»$ Werkgeschichte $\ll$. Welche Handlungen das Lesen tatsächlich nach sich zog, kommt dementsprechend allein hinsichtlich solcher Leser in Betracht, die zugleich Autoren waren und durch Bildungsromane zum Schreiben weiterer Bildungsromane veranlasst wurden. Diese Texte weichen nicht lediglich von den programmatischen Konzeptionen der Gattung ab. Sie leisten eine poetische Selbstreflexion, die nicht selten als Kritik einer idealistischen Ideologie ausgelegt wird, steht der Zielvorstellung harmonischer Bildung damit doch die literarische Darstellung gescheiterter Versuche gegenüber. Selbst Goethes vorbildlicher Held, das hat Kurt May bereits 1957 unter dem fragenden Titel »>Wilhelm Meisters Lehrjahre<, ein Bildungsroman?« dargelegt, vermag seine Leitidee ja nicht zu verwirklichen. ${ }^{130}$ Schon für die ersten Leser des Romans war es »ein zarter und heikeligter Umstand, daß er, in der Person des Meister, weder mit einer entschiednen Individualität noch mit einer durchgeführten Idealität schließt «. ${ }^{131}$ Geradezu als »Antibildungsroman« wird vielfach Karl Philipp Moritz' Anton Reiser bezeichnet, obwohl auch die Titelfigur dieses Romans eben jener utopischen Ganzheitsvorstellung anhängt, die der Neuhumanismus propagierte. ${ }^{132}$

Und dennoch, betrachtet man die solchermaßen divergenten Rezeptions- und Werkgeschichten dieser »unerfüllten Gattung « ${ }^{133}$ im Zusammenhang, lässt sich die »funktionale Zuschreibung von Bildungsroman und bewußtseinsbildender Sinnstiftung im deutschen Bürgertum ${ }^{134}$ wohl aufrechterhalten, kann sie sich doch auf die poetologischen Quellen beziehen, ohne die dort explizierten Zwecke mit den wirklichen Lektürefolgen gleichsetzen zu müssen: Leser, die das Bildungsideal bejahten und eine allseitige Entfaltung ihrer Fähigkeiten aktiv anstrebten, konnten sich anhand der Romane mit den widrigen Zwängen der Sozialisation gedanklich vertraut machen, ja womöglich schon auf halber Strecke von Wilhelm - »da ich aber nur ein Bürger bin« - lernen, Verzicht zu leisten; die Schauspielerei führt am Ende ja nicht zum Ziel. ${ }^{135}$ Der Bürger sollte »einzelne Fähigkeiten ausbilden, um brauchbar $\mathrm{zu}$ werden, und es wird schon vorausgesetzt, daß in seinem Wesen keine Harmonie sei, noch sein dürfe, weil er, um sich auf eine Weise brauchbar zu machen, alles übrige vernachlässigen muß «. ${ }^{136}$ Dagegen aber hält der Bildungsroman den Anspruch auf allseitige Selbstentfaltung aufrecht - und zwar im Einklang mit einem gesellschaftlichen Gebot, das, wie wir heute wissen, dem utilitaristischen nicht unbedingt

\footnotetext{
129 Voßkamp (Anm. 61), 345.

130 Kurt May, »Wilhelm Meisters Lehrjahre, ein Bildungsroman?«, DVjs 31 (1957), 1-37.

131 Friedrich Schiller, Brief an Goethe vom 28.11.1796, in: Der Briefwechsel zwischen Schiller und Goethe, Bd. 1: Briefe der Jahre 1794-1797, hrsg. Siegfried Seidel, München 1984, 269-271, hier: 271.

132 Vgl. Eva Blome, »Zerstückte Laufbahn. Karl Philipp Moritz' Anton Reiser«, IASL 41/2 (2016), 271-289.

133 Jürgen Jacobs, Wilhelm Meister und seine Brüder. Untersuchungen zum deutschen Bildungsroman, München 1972, 271.

134 Voßkamp (Anm. 61), 349.

135 Vgl. Heide Volkening, »Ausbildung des Charakters. Wilhelm Meisters Lehrjahre (mit Blanckenburg)«, IASL 41/2 (2016), 290-303.

136 Goethe, Wilhelm Meisters Lehrjahre (Anm. 114), 289.
} 
widerspricht. Die jüngere Auseinandersetzung mit dem so genannten »Neoliberalismus« hat vielmehr zu der Einsicht geführt, dass Individualisierung die ökonomische Nützlichkeit von Menschen nicht mindert, sondern erhöht. Kultursoziologischen Zeitdiagnosen zufolge zählt Selbstentfaltung heute zu den leitenden Werten einer neuen, aufstrebenden Mittelklasse, ${ }^{137}$ die weltweit anzutreffen ist.

\section{V.}

Die neoinstitutionalistische Theorie der Weltkultur beinhaltet eine aus der Organisationsforschung stammende Distinktion, die der Unterscheidung zwischen Charter und Funktion wie auch der zwischen manifesten und latenten Funktionen in einem gewissen Maße ähnelt, mit diesen aber nicht verwechselt werden sollte. Und zwar wird die formal-rationale Struktur institutionalisierter Akteure von ihrer alltäglichen Aktivitätsstruktur unterschieden. Dabei weist man der Ersteren eine legitimierende Funktion zu, darauf zielt die Rede von »myth« und »ceremony«. Doch klaffen organisationale Formalstrukturen und tatsächliche Abläufe nicht so weit auseinander wie die erklärte und die tatsächliche Funktion eines Regentanzes etwa. Sie sind vielmehr »lose gekoppelt«, das heißt: Die Ersteren leiten die Letzteren in begrenztem Maße an. Vor allem demonstrieren Organisationen in ihrer Außendarstellung Konformität im Verhältnis zur gesellschaftlichen Umwelt. Ebendies, das ist der springende Punkt, trägt in erheblichem Maße zu ihrem Fortbestand bei. ${ }^{138}$ So gefährdet auch ein dauerhaftes Verfehlen erklärter Ziele nicht notwendig ihre Existenz. Durch diese Akzentsetzung hebt sich der Neoinstitutionalismus von einer funktionalistischen Betrachtungsweise ab und ebenso von derjenigen Webers, wenn dessen Beschreibung der Bürokratie denn besagen soll, dass diese Herrschaftsform ihre Legitimität einer hohen Leistungsfähigkeit verdankt, die in formaler Rationalität ursächlich begründet ist. ${ }^{139}$

Das Theorem einer losen Verbundenheit von Darstellung und Performanz ist zentral für die Theorie der Weltkultur, macht es doch erklärlich, wie institutionelle Muster eine weltweite Verbreitung finden können. Dominante Akteure, so etwa bestimmte »führende« Nationalstaaten, werden imitiert, was eine formale Homogenisierung befördert und zu institutioneller Isomorphie auf der Schauseite führt. Nicht zuletzt an »Übergangsgesellschaften« und »Entwicklungsländern« ist aufgezeigt worden, dass die hinter den Modernisierungsfassaden unsichtbaren Aktivitätsstrukturen des Alltags den lokalen Gegebenheiten angepasst sind. Das macht

\footnotetext{
137 Vgl. Andreas Reckwitz, Das Ende der Illusionen. Politik, Ökonomie und Kultur in der Spätmoderne, Berlin 2020, 90-96.

138 John W. Meyer, Brian Rowan, »Institutional organizations: formal structure as myth and ceremony«, American Journal of Sociology 83 (1977), 340-363.

139 »Die rein bürokratische, also: die bürokratisch-monokratische aktenmäßige Verwaltung ist nach allen Erfahrungen die an Präzision, Stetigkeit, Disziplin, Straffheit und Verläßlichkeit, also: Berechenbarkeit für den Herren wie für die Interessenten, Intensität und Extensität der Leistung, formal universeller Anwendbarkeit auf alle Aufgaben, rein technisch zum Höchstmaß der Leistung vervollkommenbare, in all diesen Bedeutungen: formal rationalste Form der Herrschaftsausübung. « Max Weber, Wirtschaft und Gesellschaft. Grundriß der verstehenden Soziologie [1921/1922], Tübingen 1972, 128.
} 
einen Umbau der Theorie institutioneller Bedürfnisbefriedigung erforderlich, wie sich bereits in Gehlens Urmensch und Spätkultur abzeichnet. Am Beispiel der demokratischen Staatsform wird hier aufgezeigt, dass »Institutionen wie Kulturgüter, und mit ihnen ganze Sätze von normierten Verhaltensregeln [wandern] «. ${ }^{140}$ Gehlen konnte in dieser Sache sein Theorem einer Verselbstständigung der Zwecke heranziehen. Durch Habitualisierung wird Verhalten demnach formalisiert und eigenstabil. Die Gewohnheit entlastet es nicht nur von subjektiven Motiven, die Institution wird überdies unabhängig von jenen Bedürfnissen, deren Befriedigung ihr ursprünglicher Zweck einmal gewesen sein mag. Sie kann dann - auch unter Beibehaltung der Charter, wie man mit Malinowski hinzusetzen könnte, - zum Selbstzweck, oder auch umgenutzt, also in den Dienst ganz anderer Zwecke gestellt werden. Mit Merton würde ein kontingenztheoretischer Funktionalismus hier von nicht antizipierten Konsequenzen sprechen. Nach Meyer ist, wie gesagt, eine lose Kopplung anzunehmen.

Was könnte die Literaturwissenschaft dem abgewinnen? Die neoinstitutionalistische Unterscheidung ist geeignet, die Eingebundenheit von Gattungen in soziale Institutionengefüge zu beleuchten. Mit der formalen Organisation, dem autonomen Individuum oder dem souveränen Nationalstaat ist der Bildungsroman insofern vergleichbar, als es sich auch dabei um ein weltweit legitimes Kulturmuster handelt, das zur Wiederholung bereitsteht. Auch lässt sich sagen, dass vor allem seine Poetologie die Konformität mit einer gesellschaftlichen Umwelt gewährleistet, in der Individualisierung und Bildung institutionalisiert sind. Man könnte sogar die These vertreten, dass die Literatur sich in diesem Fall ihrem Umfeld nicht lediglich angepasst, sondern die Entstehung der Institutionen stark befördert, wenn nicht initiiert hat. Anstatt die idealistische Poetologie einer bürgerlichen Ideologie zuzuschlagen und die Romane, besonders die vermeintlichen »Antibildungsromane «, als deren Kritik zu loben, wäre auch hier von einer losen Kopplung auszugehen, der sich sowohl die hohe Variabilität als auch die globale Mobilität des Erzählmusters verdanken.

Der Maxime gemäß, bei der Untersuchung von Globalisierungsprozessen auf Institutionenbündel zu achten, wäre $\mathrm{zu}$ fragen, ob sich die literarische Strukturform mit jenen des Individuums, des Nationalstaats und der Organisation gemeinsam verbreitet hat. Eine mögliche Verbundenheit mit den ersteren beiden liegt auf der Hand: Das Konzept der Bildung war eine Reaktion auf die von Rousseau konstatierte Unvereinbarkeit von homme und citoyen, zwischen dem unverwechselbaren autonomen Subjekt und dem Menschen als einem sozialen, einer politischen Ordnung angehörigen Lebewesen. Die Auseinandersetzung des Individuums mit der Gesellschaft, davon handelt der optimistische Bildungsroman, sollte idealerweise zu einem harmonischen Ausgleich führen. Einen Schritt weiter geht die Forderung, die gesellschaftliche Ordnung solle der freien Entfaltung des Einzelnen günstig sein. Unter dieser Voraussetzung unternahm es Wilhelm von Humboldt, »die Grenzen der Wirksamkeit des Staats« zu bestimmen: »Der höchste und letzte Zwek jedes Menschen ist die höchste und proportionierlichste Ausbildung seiner Kräfte in ihrer

140 Arnold Gehlen, Urmensch und Spätkultur [1956], Wiesbaden 1986, 85. 
individuellen Eigenthümlichkeit «. ${ }^{141}$ Dieser Gedanke fand sehr wohl auch in anderen Ländern Anklang. John Stuart Mill zitierte den Deutschen im Epigraf seiner Schrift On Liberty mit den Worten: »The grand, leading principle, towards which every argument unfolded in these pages directly converges, is the absolute and essential importance of human development in its richest diversity. «142 Und auch Matthew Arnold, der im viktorianischen England die Idee einer Allgemeinbildung propagierte, erinnerte daran, dass »Wilhelm von Humboldt, one of the most beautiful souls that have ever existed, used to say that one's business in life was first to perfect one's self by all the means in one's power «. ${ }^{143}$

Die soziale Integration des Menschen als Bürger ist seit dem 18. Jahrhundert zu einem weltweiten Problem geworden. Nach dem Wiener Kongress hat das Modell des souveränen Nationalstaats eine starke Verbreitung erfahren. Heute stellt es eine nahezu alternativlose Form des Politischen dar. Damit zusammenhängend verbreitete sich ein Konglomerat von Mitgliedschaftsrechten und -pflichten. Im 20. Jahrhundert beförderte ein globales Regime der Menschenrechte die Verbreitung jener liberalen Konzeption des Staats, die seine Funktion auf die Sicherheitsgewährleistung beschränkt und die Bildung des Individuums zu einem seiner vornehmsten Zwecke erklärt. Mit der Charta der Vereinten Nationen des Jahres 1945 beginnend, über die politisch wegweisende, rechtlich aber nicht bindende Allgemeine Erklärung der Menschenrechte von 1948 und zahlreiche völkerrechtliche Verträge, die ab den 1960er Jahren geschlossen wurden, erstreckt sich der Prozess ihrer gesellschaftlichen Verankerung bis in die Gegenwart.

Aus neoinstitutionalistischer Sicht sind die Menschenrechte beispielhaft für ein politisches System der Weltgesellschaft, in dem Nationalstaatlichkeit als Erwartung an lokale Zusammenhänge politischen Handelns gerichtet wird. Von dieser globalen Institution geht ein Anpassungsdruck auf sämtliche Länder aus, besonders auf jene der »Dritten Welt«, die um Legitimität ringen. ${ }^{144}$ Die Menschenrechte statten Individuen mit subjektiven Rechten aus, die universelle Geltung beanspruchen, und auferlegen den Staaten zugleich Achtungs-, Schutz- und Gewährleistungspflichten. Unter anderem berühren sie damit Belange der Erziehung und Bildung, eben jene Prozesse also, von denen Romane erzählen.

Welche Dynamik die Verbreitung des Bildungsromans in der zweiten Hälfte des 20. Jahrhunderts entfaltete, bleibt noch zu erforschen. Jedenfalls war das 1956 mit der Dekolonisation Afrikas beginnende Jahrzehnt neben der Zerfallsphase der Sowjetunion einer der beiden Zeitabschnitte, für die quantitativ eine besonders starke

\footnotetext{
141 Wilhelm von Humboldt, »Ideen zu einem Versuch, die Gränzen der Wirksamkeit des Staates zu bestimmen « [1792], in: Ders., Werke in 5 Bänden, hrsg. Andreas Flitner, Klaus Giel, Bd. 1: Schriften zur Anthropologie und Geschichte, Darmstadt 1969, 64.

142 John Stuart Mill, »On Liberty«, in: Ders., On Liberty and Other Writings, hrsg. Stefan Collini, Cambridge 1989, 1-116, hier: 3 .

143 Matthew Arnold, »Culture and Anarchy«, in: Ders., Culture and Anarchy and Other Writings, hrsg. Stefan Collini, Cambridge 1993, 53-211, hier: $123 \mathrm{f}$.

${ }^{144}$ Nicht zufällig ist die Sprache der Menschenrechte in den Verfassungen dieser jüngeren Staaten besonders prominent. Vgl. Colin J. Beck, Gili S. Drori, John W. Meyer, »World Influences on Human Rights Language in Constitutions: A Cross-National Study«, International Sociology 27 (2012), 483-501.
} 
Zunahme der Nationenbildung aufgezeigt werden kann. ${ }^{145}$ Vor dem Hintergrund des bisher Ausgeführten steht zu vermuten, dass in vielen Ländern der Welt Bedürfnisse entstanden, zu deren Befriedigung die Erzählform geeignet ist. Eine Prüfung dieser Vermutung würde umfangreiche Untersuchungen erfordern. Stattdessen folgt hier zum vorläufigen Abschluss ein kurzes Referat, das auf eine Typik unterschiedlicher Funktionalisierungen der Form aus ist.

Bei erster Sondierung der vorliegenden Forschung zum postkolonialen Bildungsroman zeigt sich wiederum die bereits angesprochene Neigung, eine bestimmte Realisierung des generischen Funktionspotenzials dominant zu setzen. Die einschlägigen Studien sind in hohem Maße einem Deutungsmuster verpflichtet, das in den 1990er Jahren aufkam. Es beruht auf einer Überzeugung, die in dem wegweisenden Band The Empire Writes Back explizit gemacht wurde: »A characteristic of dominated literatures is an inevitable tendency towards subversion, and a study of the subversive strategies employed by post-colonial writers would reveal both the configurations of domination and the imaginative and creative responses to this condition. ${ }^{146}$ So komme es an der kolonialen »Peripherie« zu einer Auseinandersetzung mit den aus den imperialen »Zentren« stammenden Formen, die auf dem Wege des writing back destabilisiert würden. In jedem Falle wird die imitative Übernahme von Gattungen diesem Interpretationsschema zufolge in kritischer Absicht vollzogen. Das wollte man auch für den Bildungsroman geltend machen: »One of the reasons why postcolonial writers turn to the Bildungsroman «, so formuliert etwa José Vázquez, »is the desire to incorporate the master codes of imperialism into the text, in order to sabotage them more effectively «. ${ }^{147}$

Bedenkt man die angesprochene Funktionalisierung der Literatur durch die britische Kolonialverwaltung, scheint die Annahme, dass sich im kolonialen System ausgebildete Autoren nach der Dekolonisation von den oktroyierten Kulturmustern befreien wollten und nachfolgende Generationen dieses Ziel weiterverfolgten, nachgerade zwingend. Lima beschreibt die Situation solcher Autoren hegemonietheoretisch: Die Schwierigkeit kulturellen Widerstands bestehe darin, dass die Unterdrückten die Ideologie ihrer Unterdrücker internalisierten und reproduzierten, ihrer Unterdrückung mithin selbst zustimmten. Der Rückgriff auf die Form des Bildungsromans sei insofern »paradox«, als er einen kulturellen Imperialismus befördere, der die literarischen Akteure von der Kultur ihrer Herkunft trenne und entferne. ${ }^{148}$ Jedoch erschöpfe sich die Aneignung fremder Kulturmuster nicht in einer bloßen Annahme des Gegebenen: »>Marginal< societies are not the passive recipients of conventions and images, and their writing dismantles conventional notions of genre

\footnotetext{
145 Andreas Wimmer, Yuval Feinstein, »The rise of the nation-state across the world, 1816 to 2001 «, American Sociological Review 75/5 (2010), 764-790, hier: 784.

146 Bill Aschroft, Gareth Griffiths, Helen Tiffin, The Empire Writes Back: Theory and Practice in Postcolonial Literatures, London 1989, 33.

147 José Santiago Fernández Vázquez, »Recharting the Geography of Genre: Ben Okri's The Famished Road as a Postcolonial Bildungsroman «, Journal of Commonwealth Literature 37/2 (2002), 85-106, hier: 86. Siehe v. a. Ders., »Subverting the Bildungsroman in Post-colonial Fiction«.
}

148 Vgl. Lima (Anm. 103), 434. 
since it moves between center and margins. $\ll^{149}$ Die literarische Auseinandersetzung mit den Mustern einer hegemonialen Kultur ist demnach als ein kreativer Versuch der Selbstbefreiung postkolonialer Autoren zu würdigen, dessen Wirkung nicht auf die Peripherie beschränkt bleibt; »their literature also destabilizes the conventions of identity traditionally found in the culture of the first world «. ${ }^{150}$

So überzeugend diese Beschreibung eines Transkulturationsprozesses zunächst wirken mag: Mittlerweile haben sich die Grenzen eines vorgefertigten Deutungsmusters gezeigt, das invariant eine Dichotomie von Unterdrückten und Unterdrückern voraussetzt und alles Schreiben mit einer traumatischen Erfahrung der Kolonisation in Verbindung bringt. ${ }^{151}$ Auch was den Bildungsroman anbelangt, wird neuerdings davon abgeraten, ihn pauschal als reaktive, im Dienst antikolonialer Politik stehende Form anzusehen. ${ }^{152}$ Sehr wahrscheinlich war und ist die Bedürfnislage der Literaturschreibenden und -lesenden in vormals kolonisierten Ländern komplex. Mithin gilt es zumindest die Möglichkeit in Betracht zu ziehen, dass die überdeterminierte Institution auch andere Funktionen erfüllt. Nicht zuletzt scheint die Festlegung auf eine gegendiskursive Funktion deshalb fragwürdig, weil sie sich einseitig an der idealistischen Programmatik orientiert - dass die literarischen Texte seit jeher in einem spannungsvollen Verhältnis zur leitenden Bildungsidee stehen, bleibt außer Acht.

Unter Berücksichtigung dieser Spannung hat Horst Nitschak in seinen Arbeiten zum lateinamerikanischen Bildungsroman eine alternative Lesart erprobt: Mit der Erzählform sei zugleich ein Modell des Individuums und der Individualisierung aus Europa exportiert worden. Die Qualitäten des selbstständigen, verantwortlichen, moralisch handelnden und freien Individuums, nach denen die Helden solcher Romane strebten, würden vermittels der Literatur auch in anderen Teilen der Welt verbreitet. ${ }^{153}$ Halten wir das fest: Wenn postkoloniale Romane von den Schwierigkeiten oder vom Scheitern dementsprechender Versuche erzählen, unterscheiden sie sich darin kaum von den europäischen Mustertexten. Eine Besonderheit der kulturellen Situation Lateinamerikas und des globalen Südens allgemein ist mit Nitschak allerdings darin zu erkennen, dass jene Werte und Ideale, die von den vorgeblich ehrwürdigen Eliten propagiert werden, nicht aus der eigenen Lebenswelt hervorgegangen, sondern aus den nördlichen Metropolen eingeführt worden sind. ${ }^{154}$ Auch in dieser Situation, das ist entscheidend, werden »Bildung « und »Individualisierung «

\footnotetext{
149 Lima (Anm. 103), 455.

150 Lima (Anm. 103), 455.

151 Vgl. Ankhi Mukherjee, What is a Classic? Postcolonial Rewriting and Invention of the Canon, Stanford 2014, 116.

152 Ericka A. Hoagland, »The Postcolonial Bildungsroman«, in: Sarah Graham (Hrsg.), A History of the Bildungsroman, Cambridge 2019, 217-238, hier: 227.

153 Horst Nitschak, »Postkoloniale Subjektivität: Macunaíma, ein Held ohne jeden Charakter (Mário de Andrade). Ein Bildungsroman in den Tropen?«, in: Martina Kopf, Sascha Seiler (Hrsg.), Komparatistische Blicke auf Lateinamerika und Europa. Intercultural Studies. Schriftenreihe des Zentrums für Interkulturelle Studien (ZIS), Heidelberg 2016, 171-184, hier: 175.

154 Horst Nitschak, »Tropische Subjektivität und europäische Bildungstradition: Macunaíma, der Held ohne jeden Charakter von Mário de Andrade. Oder: Macunaíma, ein Wilhelm Meister in den Tropen?« Pandaemonium Germanicum 16/22, Sao Paulo 2013, 156-178, hier: 177.
} 
aber gesellschaftlich erwartet. Generell nahmen die sich im Laufe des 19. Jahrhunderts konsolidierenden Nationen Lateinamerikas ja die Ursprungsländer der Romanliteratur zum Vorbild ihrer kulturellen und politischen Entwicklung und machten sich Strukturformen bündelweise zu eigen. ${ }^{155}$ So überrascht es nicht, dass sich einem komparativen Blick auf das deutsche Erzählmuster und den Roman Macunaíma (1928) des Brasilianers Mário de Andrade erweist, »dass unter vergleichbaren historischen Herausforderungen, wie der Herausbildung eines Nationalstaates und der Suche nach einer subjektiven Moralität, die das Individuum gleichzeitig zu einem verantwortungsbewussten >citizen< (cidadão) werden lässt, vergleichbare, wenn auch in sich wieder sehr unterschiedliche Antworten gegeben werden « ${ }^{156}$

Wie Joseph Slaughter deutlich gemacht hat, finden sich in der postkolonialen Literatur sehr wohl Texte, die sich einem Typus des »idealist (or affirmative) Bildungsroman « zuordnen lassen, ${ }^{157}$ als dessen Muster er Goethes Lehrjahre ansieht, verstanden als optimistische Darstellung individueller Entfaltung. Darin konvergiert das literarische Erzählen mit der Zuerkennung eines Anspruchs auf freie Persönlichkeitsentfaltung durch die Menschenrechte. Slaughter räumt ein, dass diese im 20. Jahrhundert zum zentralen Bestandteil einer liberal-demokratischen Ideologie wurden, weist jedoch auf eine nicht antizipierte Folge hin, nämlich die »appropriation and transformative rearticulation of the egalitarian imaginary by historically marginalized subjects (e.g., women and members of racial, religious, sexual, and class minorities) not comprehended practically within its original enabling fiction $\ll .{ }^{158} \mathrm{Bil}-$ dung wird demnach von manchen Schriftstellerinnen und Leserinnen nicht als hegemoniale Direktive, sondern als eine befähigende Leitvorstellung aufgefasst. Auch in vormals kolonisierten Gesellschaften wird diese mitunter bejaht. Darin ist eine Funktion des Bildungsromans zu erkennen. Slaughter fragt ausdrücklich nach »the genre's social function «: ${ }^{159}$ ein weiterer Beleg für das Fortdauern der funktionalistischen Denkweise.

\section{VI.}

Eine theoriegeschichtliche Reflexion, das mag das Vorstehende immerhin deutlich gemacht haben, könnte der Literaturwissenschaft $\mathrm{zu}$ einem erhöhten Bewusstsein von den teils problematischen Implikationen verhelfen, die mit einer Funktionsgeschichte literarischer Institutionen verbunden sind, wie auch bei der Suche nach Problemlösungen orientierend wirken. Eine neoinstitutionalistische Aktualisierung älterer Ansätze würde zudem einen Blick auf transnationale Prozesse eröffnen, deren Dynamik sich im zurückliegenden Jahrhundert stark verändert hat - in zuneh-

\footnotetext{
155 Vgl. Horacio Mansilla, »Die Konfrontation mit der bereits entwickelten Welt und die Suche nach eigenen Modellen in Lateinamerika«, in: Josef Thesing (Hrsg.), Lateinamerika. Tradition und Modernität, Mainz 1991, 83-97.

156 Nitschak (Anm. 154), 173.

157 Slaughter (Anm. 62), 93.

158 Slaughter (Anm. 62), 5.

159 Slaughter (Anm. 62), 7.
} 
mendem Maße ist auch Literatur Teil eines größeren Ganzen, auf das der Begriff $»$ Weltgesellschaft«, wie unzureichend auch immer, hindeutet.

Ein letztes Problem sei nochmals angesprochen, das Problem der Selbstimplikation: Schelsky hat Theorien im Hinblick darauf sortiert, und dafür haben wir viele Beispiele gesehen, dass sie Institutionen teils »vom Ganzen der Gesellschaft«, teils »vom gesellschaftlichen Handeln her « betrachten. ${ }^{160}$ Diese Gegensätzlichkeit der Gesichtspunkte führe »bei denkkonsequenter Verfolgung des Theorieansatzes zu antagonistischen Problem- und Kategoriensystemen, [...] so entstehen die endlos diskussionsfähigen, aber unlösbaren sozialwissenschaftlichen Probleme. ${ }^{161}$ Die eingangs angesprochene Vieldeutigkeit des Begriffs der Institution hängt wohl damit zusammen. Möglicherweise handelt es sich dabei nicht um einen behebbaren Mangel, sondern um die kennzeichnende Eigenschaft eines geschichtlichen Grundbegriffs, der wesentlich umstritten ist. Wissenschaftliche Institutionentheorien wären dann auch als mitunter gegensätzliche Stellungnahmen zu einem gesellschaftlichen Problem zu verstehen. Wenn es zutrifft, dass die Philologie im Verbund mit dem Bildungsroman eine bestimmte Weise der Subjektivierung, nämlich Individualisierung, vorangetrieben hat, dann ist sie in dieser Streitsache vermutlich voreingestellt, und zwar gegen Betrachtungen vom Ganzen, erst recht vom Weltganzen her, die bewusst nicht die Perspektive der institutionellen Akteure einnehmen. Es wäre also $\mathrm{zu}$ prüfen, ob die Literaturwissenschaft einem methodologischen Individualismus zuneigt, der zur Verfestigung eines hegemonialen Kulturmusters beiträgt, »driving the >cult of individualism < to heights Durkheim could never have envisioned «. ${ }^{162}$

Funding Open Access funding provided by Projekt DEAL.

Open Access Dieser Artikel wird unter der Creative Commons Namensnennung 4.0 International Lizenz veröffentlicht, welche die Nutzung, Vervielfältigung, Bearbeitung, Verbreitung und Wiedergabe in jeglichem Medium und Format erlaubt, sofern Sie den/die ursprünglichen Autor(en) und die Quelle ordnungsgemäß nennen, einen Link zur Creative Commons Lizenz beifügen und angeben, ob Änderungen vorgenommen wurden.

Die in diesem Artikel enthaltenen Bilder und sonstiges Drittmaterial unterliegen ebenfalls der genannten Creative Commons Lizenz, sofern sich aus der Abbildungslegende nichts anderes ergibt. Sofern das betreffende Material nicht unter der genannten Creative Commons Lizenz steht und die betreffende Handlung nicht nach gesetzlichen Vorschriften erlaubt ist, ist für die oben aufgeführten Weiterverwendungen des Materials die Einwilligung des jeweiligen Rechteinhabers einzuholen.

Weitere Details zur Lizenz entnehmen Sie bitte der Lizenzinformation auf http://creativecommons.org/ licenses/by/4.0/deed.de.

160 Schelsky (Anm. 13), 10 (Hv. i. O.).

161 Helmut Schelsky, »Systemfunktionaler, anthropologischer und personfunktionaler Ansatz in der Rechtssoziologie«, in: Ders, Die Soziologen und das Recht (Anm. 43), Opladen 1980, 95-146, hier: 98.

162 Ronald Jepperson, John W. Meyer, »Multiple Levels of Analysis and the Limitations of Methodological Individualism«, Sociological Theory 29/1 (2011), 54-73, hier: 69. 